\title{
Native defect-induced multifarious magnetism in nonstoichiometric cuprous oxide: First-principles study of bulk and surface properties of $\mathrm{Cu}_{2-\delta} \mathrm{O}$
}

\author{
Aloysius Soon, ${ }^{1, *}$ Xiang-Yuan Cui, ${ }^{1}$ Bernard Delley, ${ }^{2}$ Su-Huai Wei, ${ }^{3}$ and Catherine Stampfl ${ }^{1}$ \\ ${ }^{1}$ School of Physics, The University of Sydney, Sydney, NSW 2006, Australia \\ ${ }^{2}$ Paul-Scherrer-Institut, WHGA/123, CH-5232, Villigen PSI, Switzerland \\ ${ }^{3}$ National Renewable Energy Laboratory, 1617 Cole Boulevard, Golden, Colorado 80401, USA
}

(Received 25 September 2008; published 26 January 2009)

\begin{abstract}
Native defects in cuprous oxide $\mathrm{Cu}_{2} \mathrm{O}$ are investigated by using first-principles calculations based on density-functional theory. Considering the formation of copper and oxygen vacancies, antisites and interstitials, and a copper split-vacancy complex defect, we analyze the electronic structure and calculate their respective formation energies as a function of the change in Fermi level under both copper-rich and oxygen-rich conditions. We find that, under both growth conditions, the defect with the lowest formation energy is the simple copper vacancy, followed by the copper split-vacancy complex. Both low-energy copper defects produce hole states at the top of the valence band, largely accounting for the $p$-type conductivity in this material. In spite of the creation of dangling bonds at the nearest-neighbor $\mathrm{O}$ atoms, these copper defects are found to be spin neutral. Under oxygen-rich conditions, oxygen interstitials have low formation energies and are found to exhibit a ferromagnetic ordering with a total magnetic moment of $1.38 \mu_{B}$ and $1.36 \mu_{B}$ at the octahedral and tetrahedral sites, respectively. Considering the possibility of native defect formation at the surface of this material, we investigate the relative stability of both low- and high-index copper-oxide surfaces by comparing their surface free energies as a function of the change in oxygen chemical potential. Using the technique of $a b$ initio atomistic thermodynamics, we then correlate the dependence of the calculated Gibbs free-surface energy as a function of oxygen pressure and temperature via the oxygen chemical potential. We identify two lowenergy surface structures, namely, $\mathrm{Cu}_{2} \mathrm{O}(110): \mathrm{CuO}$ and $\mathrm{Cu}_{2} \mathrm{O}(111)-\mathrm{Cu}_{\mathrm{CUS}}$, with the former marginally more stable for oxygen-rich conditions and the latter more stable for oxygen-lean (or copper-rich) conditions. $\mathrm{Cu}_{2} \mathrm{O}(110): \mathrm{CuO}$ is calculated to be nonmagnetic and $\mathrm{Cu}_{2} \mathrm{O}(111)-\mathrm{Cu}_{\text {CUS }}$ is calculated to be a ferromagnetic ordering, with a total magnetic moment of $0.91 \mu_{B}$ per defect. With the results for both bulk and surface native defects, we find that under oxygen-lean conditions, a ferromagnetic behavior could be attributed mainly to copper vacancy formation in the (111) surface of $\mathrm{Cu}_{2} \mathrm{O}$ while under oxygen-rich conditions, low-energy bulk oxygen interstitial defects induce a ferromagnetic character in the same material. This highlights the complementary role of bulk and surface native magnetic defects under different pressure and temperature conditions, especially at the nanoparticle scale where surface properties dominate.
\end{abstract}

DOI: 10.1103/PhysRevB.79.035205

PACS number(s): 71.55.-i, 71.20.-b, 72.20.Jv, 72.80.Jc

\section{INTRODUCTION}

The catalytic and semiconducting properties of cuprous oxide $\mathrm{Cu}_{2} \mathrm{O}$ have recently been studied with great interest (see e.g., Refs. 1-8 and references therein). With regards to its catalytic properties, the $\mathrm{O} / \mathrm{Cu}$ system (i.e., oxidic nanostructures of $\mathrm{Cu}$ ) is known to facilitate oxidation reactions, suggesting that it has potential as an economical alternative for the rare and high-cost noble metals in various catalytic systems. $^{3-5,9-12}$ Our recent study on the $\mathrm{O} / \mathrm{Cu}(111)$ system $^{13}$ has predicted that, under technical catalytic conditions of gas pressures and temperatures, the thermodynamically favored phase is that of the bulk oxide. Hence the oxide $\mathrm{Cu}_{2} \mathrm{O}$ should be considered as one of the key active catalytic phases in technologically relevant reactions. Regarding its semiconducting properties, its $p$-type conductivity arises from the existence of holes in the valence band (VB). ${ }^{1,7,14}$ With an increased interest in the design of transparent electronics using $p$-type conductive oxides, it is found that by alloying $\mathrm{Cu}_{2} \mathrm{O}$ with other metal oxides such as $\mathrm{SrO}$ or $\mathrm{Al}_{2} \mathrm{O}_{3}$, the resultant material is both transparent and conducting, hence making its alloys potential candidates for developing transparent conducting electronic devices. ${ }^{7}$
Concurrently, with the recent pursuit for spintronicsrelated technology, ${ }^{15,16}$ magnetic oxide semiconductors are receiving much attention as potential candidate materials of interest and promoting the rather new and exciting field of "oxide spintronics." 17-24 Oxides, unlike the III-V compounds, tend to exhibit a much higher Curie temperature when doped and can accommodate a much higher doping concentration of magnetic impurity atoms. ${ }^{24}$ More interestingly, some oxides that are traditionally thought to be "nonmagnetic" have recently been shown to exhibit relatively large intrinsic magnetic moments, i.e., without the intentional introduction of magnetic dopants. ${ }^{25-30}$ This has prompted many researchers to (re-)investigate the fundamental properties of such semiconducting oxides, focusing largely on their deviation from natural stoichiometry and the role of native defects. In relation to $\mathrm{Cu}_{2} \mathrm{O}$, Yermakov and co-workers ${ }^{31,32}$ have found that nanocrystalline powder samples of nonstoichiometric $\mathrm{Cu}_{2} \mathrm{O}$ (i.e., $\mathrm{Cu}_{2-\delta} \mathrm{O}$ ) exhibit magnetic hysteresis properties of up to $400 \mathrm{~K}$, suggesting ferromagnetic behavior. These nanoparticles are of 10-100 $\mathrm{nm}$ in size and their magnetic properties are reported to be highly dependent on the oxygen concentration during their formation. The nature and origin of magnetism observed for 
these nanoparticles are still largely unknown. The authors only suggest that the possible origin of this observed intrinsic magnetism is due to the nonstoichiometry of the sample. Native point defects and/or complexes are speculated to be responsible for the observed magnetic properties. Given these aspects and the high technological importance of copper oxide, it calls for an in-depth investigation and understanding of the behavior and role of bulk native defects, as well as the surface properties of this material. In addition, being in the same family as copper-oxide-based superconductors, having a firm knowledge of the electronic structure of this "simple" metal oxide could well shed some light on our present knowledge of their more complex "cousins"the copper-oxide-based superconductors (e.g., yttriumbarium-copper oxides).

The first part of this work will investigate the role of native defects in bulk $\mathrm{Cu}_{2} \mathrm{O}$ and their influence on the electronic structure of this material. It has been established experimentally ${ }^{33-35}$ and theoretically ${ }^{7,8,14}$ that the dominant point defects in cuprous oxide under varying oxygen conditions are both neutral and singly negatively charged copper vacancies. As reported, both the electronic and magnetic properties of $\mathrm{Cu}_{2} \mathrm{O}$ is sensitively influenced by its (sub)stoichiometry and growth conditions, ${ }^{31-34}$ thus reinforcing the need to investigate how deviation from its natural stoichiometry (i.e., in the presence of native defects) will affect these properties (and thus functionality) in $\mathrm{Cu}_{2} \mathrm{O}$. Using the projector-augmented-wave (PAW) method and the generalized gradient approximation (GGA), Nolan and Elliott ${ }^{7}$ performed density-functional theory (DFT) calculations, investigating the $p$-type conduction mechanism in $\mathrm{Cu}_{2} \mathrm{O}$, and concluded that the $p$-type semiconducting properties seen in $\mathrm{Cu}_{2} \mathrm{O}$ are attributed to small concentrations of $\mathrm{Cu}$ vacancies. Another DFT-GGA study within the same PAW framework by Raebiger et al. ${ }^{14}$ also demonstrates that $\mathrm{Cu}_{2} \mathrm{O}$ is indeed intrinsically cation deficient due to the presence of $\mathrm{Cu}$ vacancies that act as shallow and efficient hole producers. However, an in-depth study of its defect-associated magnetic properties is still lacking. Thus in this work, we then proceed to investigate the magnetic properties of both the low-energy bulk defects as well as that of low-energy surfaces of $\mathrm{Cu}_{2} \mathrm{O}$ identified in our previous study. ${ }^{36}$ In addition, we also report the energetics and magnetic properties of two high-index surfaces, namely, $\mathrm{Cu}_{2} \mathrm{O}(210)$ and $\mathrm{Cu}_{2} \mathrm{O}(311)$. Having investigated both bulk and surface properties, we then attempt to show that the multifarious magnetic behavior of this material could possibly originate from different sources under different oxygen atmospheres.

\section{METHOD AND APPROACHES}

All calculations are performed using DFT and the GGA of Perdew, Burke, and Ernzerhof (PBE) (Ref. 37) for the exchange-correlation functional as implemented in the allelectron $\mathrm{DMol}^{3}$ code. The $\mathrm{DMol}^{3}$ method employs fast converging three-dimensional numerical integrations to calculate the matrix elements occurring in the Ritz variational method. The wave functions are expanded in terms of a doublenumerical quality localized basis set with a real-space cutoff (a)

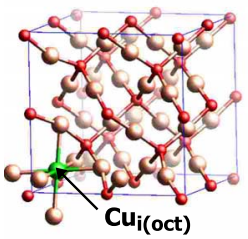

(d)

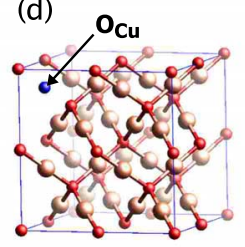

(g)

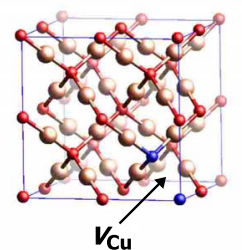

(j)

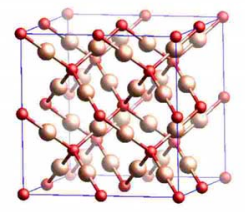

(b)

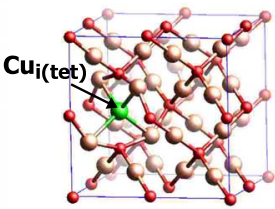

(e)

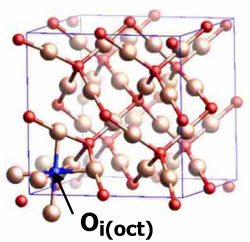

(h)

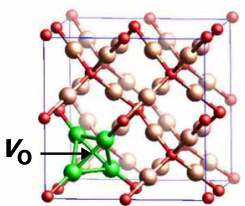

(k)

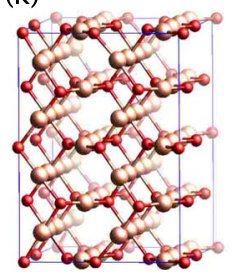

(c)

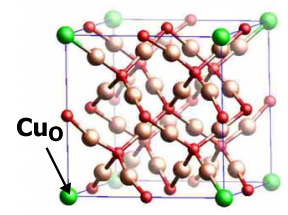

(f)

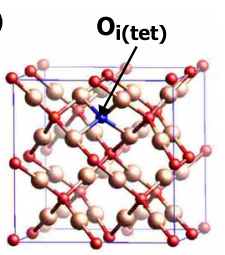

(i)

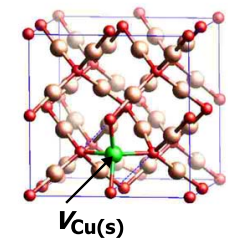

(I)

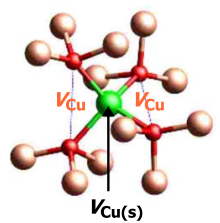

FIG. 1. (Color online) Optimized atomic geometry of native defects in the 48-atom $\mathrm{Cu}_{2} \mathrm{O}$ supercell: (a) $\mathrm{Cu}_{i \text { (oct) }}$, (b) $\mathrm{Cu}_{i \text { (tet) }}$, (c) $\mathrm{Cu}_{\mathrm{O}}$, (d) $\mathrm{O}_{\mathrm{Cu}},\left(\right.$ e) $\mathrm{O}_{i \text { (oct) }}$, (f) $\mathrm{O}_{i \text { (tet) }}$, (g) $V_{\mathrm{Cu}}$, (h) $V_{\mathrm{O}}$, and (i) $V_{\mathrm{Cu}(\mathrm{S})}$. (j) and $(\mathrm{k})$ shows the stoichiometric 48-atom and 96-atom $\mathrm{Cu}_{2} \mathrm{O}$ supercells, respectively. (1) depicts the local environment of the $\mathrm{Cu}$ split vacancy $V_{\mathrm{Cu}(\mathrm{s})}$ which comprises one interstitial $\mathrm{Cu}$ inserted in between two $V_{\mathrm{Cu}}$. Copper atoms are shown as large (orange) spheres, and oxygen atoms as the smaller (red) spheres.

of 9 bohr. Polarization functions and scalar-relativistic corrections are incorporated explicitly. More details of the $\mathrm{DMol}^{3}$ code can be found elsewhere. ${ }^{38,39}$ Bulk defect calculations are mainly performed using 48-atom supercells [see Fig. 1(j)], i.e., a $(2 \times 2 \times 2) a$ unit cell (where $a$ is the lattice constant). Selected convergence tests are performed using larger supercells, namely, 96- [e.g., Fig. 1(k)], 144-, and 162atom supercells, which are reported in Sec. III. The Brillouin-zone (BZ) integration is performed using Monkhorst-Pack grids of $(6 \times 6 \times 6)$ with $10 \mathbf{k}$ points in the irreducible part of the BZ for the 48-atom supercell. For the other supercells, the grids have been folded to obtain the same sampling of reciprocal space. The positions of all atoms are fully relaxed until the force acting on each atom is less than $0.3 \mathrm{mHa} / \mathrm{bohr}(0.015 \mathrm{eV} / \AA)$. These parameters were chosen to ensure that calculated energies are well converged, such that changing the $\mathbf{k}$-point grid from $(6 \times 6$ $\times 6)$ to $(7 \times 7 \times 7)$ yields no change in the formation energy of $\mathrm{Cu}_{2} \mathrm{O}$ and increasing the real-space cutoff parameter from 9 to 10 bohr changes the same quantity by less than $10 \mathrm{meV}$. 
The description of the basis set used is further elaborated on in Refs. 13 and 36. To investigate the magnetic properties of the considered defects, all calculations are performed employing spin polarization in the 48 -atom supercell. To simulate an antiferromagnetic solution, we use the 96-atom supercell (i.e., double the 48-atom supercell in the $c$ direction) and enforce opposite spins on adjacent defects in the same cell.

The formation energy ${ }^{40} \Delta H^{f}(q)$ of a native defect in charge state $q$ (i.e., $q$ takes a positive number when electrons are transferred from the system to the reservoirs in forming the defect, and vice versa) is expressed as

$$
\begin{aligned}
\Delta H^{f}(D, q)= & E^{\mathrm{def}}(D, q)-E^{\mathrm{bulk}}+\sum_{i} n_{i} \mu_{i} \\
& +q\left[\Delta E_{F}+\epsilon_{\mathrm{VBM}}^{\mathrm{bulk}}+\Delta v(D)\right]
\end{aligned}
$$

where $E^{\operatorname{def}}(D, q)$ is the total energy of the supercell containing the defect $D, E^{\text {bulk }}$ is the total energy of the corresponding reference supercell containing the pure bulk crystal (i.e., without the considered defect), and $n_{i}$ and $\mu_{i}$ are the number of atoms and chemical potential of species $i$ (where $i$ is $\mathrm{Cu}$ or O), respectively. $n_{i}$ takes a positive (negative) value when the species $i$ is removed from (added to) the system. $\Delta E_{F}$ is the Fermi energy level which is set to zero at the valence-band maximum (VBM), and $\epsilon_{\mathrm{VBM}}^{\text {bulk }}$ is the VBM eigenvalue of the pure bulk crystal. For different defects, a proper lineup of the potentials of the defect supercells with that of the bulk is required. This is done by lining up the $\mathrm{O} 1 s$ core energy level $\epsilon_{\mathrm{core}, 1 s}^{\mathrm{far}}(D)$ of the furthest $\mathrm{O}$ atom from the defect location, which will have a bulklike environment with that in the bulk cell, $\epsilon_{\text {core }, 1 s}^{\text {bulk }}$, i.e., giving rise to $\Delta v(D)=\epsilon_{\text {core }, 1 s}^{\text {far }}(D)-\epsilon_{\text {core }, 1 s}^{\text {bulk }}$. With this definition, $\left[\epsilon_{\mathrm{VBM}}^{\mathrm{bulk}}+\Delta v(D)-\epsilon_{\mathrm{core}, 1 s}^{\mathrm{far}}(D)\right]$ is then set to be a constant for different defect cells. From Eq. (1), we can define the electronic transition energy, $\epsilon\left(q / q^{\prime}\right)$, at which the formation energy $\Delta H^{f}(D, q)$ of defect $D$ at charge state $q$ is equal to $\Delta H^{f}\left(D, q^{\prime}\right)$ at charge state $q^{\prime}$, i.e., ${ }^{40}$

$$
\epsilon\left(D, q / q^{\prime}\right)=\frac{E^{\operatorname{def}}(D, q)-E^{\operatorname{def}}\left(D, q^{\prime}\right)}{q^{\prime}-q} .
$$

To obtain the transition energy level $\Delta \epsilon\left(D, q / q^{\prime}\right)$ with respect to the bulk VBM, we can use the relationship

$$
\Delta \epsilon\left(D, q / q^{\prime}\right)=\epsilon\left(D, q / q^{\prime}\right)-\epsilon_{\mathrm{VBM}}^{\text {bulk }}-\Delta v(D) .
$$

To relate the formation energy to its defect concentration in the crystal, we refer to the following: ${ }^{41}$

$$
c=N_{\text {sites }} N_{\text {config }} \exp \left(-\frac{\Delta H^{f}(q)}{k_{B} T}\right) .
$$

That is, for a given temperature and in the limit of low concentrations, one can assume that defect concentrations obey an Arrhenius type of behavior, which means that the logarithms of concentrations are proportional to the formation energies. A low value of the formation energy would then indicate a high equilibrium concentration, $c$, of defects while a high value implies a low concentration. In this case, $N_{\text {sites }}$ is defined as the number of sites in the crystal lattice per unit volume that the defect can be incorporated into. For this study we assume the number of equivalent configurations, in which the defect can be incorporated $N_{\text {config, }}$ to be one.
Strictly speaking, Eq. (4) is only obeyed under thermodynamic equilibrium. Thus, considering that growth conditions are typically far from thermal equilibrium, calculated formation energies can serve as a good alternative for the discussion of defect "concentration" in the material. In this way, high-energy defects are less likely to exist in the material and the vice versa is true, as well.

The chemical potentials depend on the experimental conditions under which the material is grown. In order to determine these quantities, we invoke the relationship $2 \mu_{\mathrm{Cu}}+\mu_{\mathrm{O}}$ $=\mu_{\mathrm{Cu}_{2} \mathrm{O}}$ on the assumption that both $\mathrm{Cu}$ and $\mathrm{O}$ species are in thermal equilibrium with $\mathrm{Cu}_{2} \mathrm{O}$. In reality, again, growth conditions could well be far from equilibrium. In addition, the chemical potentials must satisfy the following boundary conditions: $\mu_{\mathrm{O}}<\frac{1}{2} \mu_{\mathrm{O}_{2}}$ and $\mu_{\mathrm{Cu}}<\mu_{\mathrm{Cu} \text { (bulk) }}$. This is to ensure that $\mathrm{Cu}_{2} \mathrm{O}$ is thermodynamically stable with respect to the formation of $\mathrm{O}_{2}$ or bulk $\mathrm{Cu}$. Under specific growth conditions, namely, under oxygen-rich and copper-rich conditions, the chemical potential of $\mathrm{O}, \mu_{\mathrm{O}}$, is defined as $\frac{1}{2} \mu_{\mathrm{O}_{2}}$ (i.e., $\Delta \mu_{\mathrm{O}}=\mu_{\mathrm{O}}-\frac{1}{2} \mu_{\mathrm{O}_{2}}=0$ ) and that of $\mathrm{Cu}, \mu_{\mathrm{Cu}}$, to be $\mu_{\mathrm{Cu} \text { (bulk) }}$ (i.e., $\left.\Delta \mu_{\mathrm{Cu}}=\mu_{\mathrm{Cu}}-\mu_{\mathrm{Cu} \text { (bulk) }}=0\right)$, respectively. In this work, we restrict ourselves to these chemical-potential limits whereby higher oxides of $\mathrm{Cu}$, namely, $\mathrm{Cu}_{4} \mathrm{O}_{3}$ (metastable) and $\mathrm{CuO}$, are not considered due to the complexity of the electronic structure of these two higher oxides and would require postDFT approaches, such as DFT $+U .^{14}$

To calculate the various $\mathrm{Cu}_{2} \mathrm{O}$ surfaces, we use surface supercells containing symmetric slabs (with inversion symmetry) with 15-19 atomic layers (ranging from 30 to 56 atoms) for the low-index surfaces. These correspond to a slab thickness of about 11, 24, and $18 \AA$ for the (111), (110), and (100) surfaces of $\mathrm{Cu}_{2} \mathrm{O}$, respectively. These slabs are considerably thicker than required for the convergence of surface properties as we investigate the formation of subsurface defects (up to the third or fourth atomic-layer deep). Typically, the surface energies of the (111), (110), and (100) surfaces of $\mathrm{Cu}_{2} \mathrm{O}$ are well converged to $\leq 10 \mathrm{meV} / \AA^{2}$ with slab thicknesses of about 8,20 , and $14 \AA$, respectively. ${ }^{36}$ In addition to these low-index terminations, we have also explicitly considered the next higher-index oxide surfaces, namely, $\mathrm{Cu}_{2} \mathrm{O}(210)$ and $\mathrm{Cu}_{2} \mathrm{O}(311)$ [see Figs. 7(c) and 7(d)]. These so-called microfaceted oxide surfaces could offer an initial step toward understanding the surface properties of polycrystalline samples of $\mathrm{Cu}_{2} \mathrm{O}$. For these two surfaces, we align the $c$ axis of the supercell in the direction of the high-index crystallographic surface, i.e., [210] and [311], respectively. Thus it is not straight forward to describe the thickness of the slabs used in atomic layers; rather we report a slab thickness of $\sim 19$ (62 atoms in the slab) and $\sim 14 \AA$ (68 atoms in the slab) for the high-index $\mathrm{Cu}_{2} \mathrm{O}(210)$ and $\mathrm{Cu}_{2} \mathrm{O}(311)$ surfaces, respectively. These slab thicknesses are comparable to those typically used for the low-indexed surfaces. For all surfaces, a 30-40 $\AA$ vacuum region is used between adjacent slabs. For the low-index surfaces, all surfaces are fully relaxed while keeping the innermost three center layers fixed at the bulk values, while for the two high-index surfaces, the innermost layers corresponding to a thickness of $\sim 6$ and $\sim 4 \AA$ for $\mathrm{Cu}_{2} \mathrm{O}(210)$ and $\mathrm{Cu}_{2} \mathrm{O}(311)$, respectively, are kept fixed. The BZ integration is performed using Monkhorst-Pack grids 
of $(8 \times 8 \times 1),(12 \times 6 \times 1)$, and $(12 \times 12 \times 1)$ with 10,18 , and $42 \mathbf{k}$ points in the irreducible part of the BZ for the (111), (110), and (100) surface unit cells, respectively. For both $\mathrm{Cu}_{2} \mathrm{O}(210)$ and $\mathrm{Cu}_{2} \mathrm{O}(311)$, a Monkhorst-Pack grid of $(8 \times 4 \times 1)$ with $16 \mathbf{k}$ points in the irreducible part of the BZ is used. For all low-index orientations we have verified that using thicker slabs does not result in any significant changes (i.e., $\leq 1 \mathrm{meV} / \AA^{2}$ for the surface energy). ${ }^{36}$ With these basis sets, the surface energies of different low-index oxide surfaces are converged to within $2 \mathrm{meV} / \AA^{2}$ regarding $\mathbf{k}$ points and real-space cutoff parameter. ${ }^{36}$ Due to the complexity of the high-index surfaces as well as our prior experience with the low-index surfaces of $\mathrm{Cu}_{2} \mathrm{O}$, we restrict ourselves to the above-mentioned slab thicknesses and $\mathbf{k}$-point grids.

\section{RESULTS AND DISCUSSION}

\section{A. Structural properties of $\mathrm{Cu}_{2} \mathrm{O}$}

$\mathrm{Cu}_{2} \mathrm{O}$ crystallizes in a cuprite structure with space-group $P n \overline{3} m .{ }^{42}$ Each $\mathrm{Cu}$ atom is linearly coordinated to two oxygen atoms, and all oxygen atoms are tetrahedrally surrounded by four $\mathrm{Cu}$ atoms. $\mathrm{Cu}_{2} \mathrm{O}$ is a direct band-gap semiconductor with a calculated DFT band gap of $0.46 \mathrm{eV}$ which is in very good agreement with other theoretical DFT-GGA values of 0.43 (Ref. 14) and $0.47 \mathrm{eV}^{7}$ The well-established experimental value ${ }^{43}$ is $2.17 \mathrm{eV}$ and typically is significantly larger than that obtained by DFT-GGA (or the local-density approximation). We found the optimized lattice constant, bulk modulus, and heat of formation for cuprous oxide to be $4.32 \AA, 103.7$ $\mathrm{GPa}$, and $1.24 \mathrm{eV}$, respectively, as previously reported. ${ }^{13}$ These are in good agreement with corresponding experimental values of $4.27 \AA$, $112 \mathrm{GPa}$, and $1.75 \mathrm{eV},{ }^{44-47}$ although the GGA underestimates the heat of formation by about $30 \%$. This can in part be attributed to the overestimation of the binding energy of the oxygen molecule. For details regarding the calculation of bulk $\mathrm{Cu}$ and $\mathrm{O}_{2}$, we refer to Refs. 13 and 36.

\section{B. Native defects in bulk $\mathrm{Cu}_{2} \mathrm{O}$}

We have considered the formation of native point defects, namely, copper $\left(V_{\mathrm{Cu}}\right)$ and oxygen $\left(V_{\mathrm{O}}\right)$ vacancies, copper $\left(\mathrm{Cu}_{\mathrm{O}}\right)$ and oxygen $\left(\mathrm{O}_{\mathrm{Cu}}\right)$ antisites, and copper $\left(\mathrm{Cu}_{i \text { (oct) }}\right.$, $\left.\mathrm{Cu}_{i \text { (tet) }}\right)$ and oxygen $\left(\mathrm{O}_{i(\text { oct })}\right.$ and $\left.\mathrm{O}_{i(\text { tet })}\right)$ interstitials at two different sites, namely, the octahedral (with subscript "oct") and tetrahedral (with subscript "tet"). The octahedral interstitial site has the interstitial atom surrounded by six nearest $\mathrm{Cu}$ atoms and likewise, for the tetrahedral site, it will be surrounded by four $\mathrm{Cu}$ atoms. An antisite is created by replacing a $\mathrm{Cu}$ atom with an $\mathrm{O}$ atom, and vice versa, i.e., an oxygen in a metal-atom site. In addition, we considered a so-called copper split-vacancy complex defect, denoted by $V_{\mathrm{Cu}(\mathrm{s})}{ }^{7,8,14}$ This defect [see Figs. 1(i) and 1(1)] can be understood as the insertion of an interstitial copper atom in between two $V_{\mathrm{Cu}}$, and hence a defect complex. This results in a local geometry distortion about the $\mathrm{Cu}$ vacancy site, $V_{\mathrm{Cu}}$, i.e., a structural displacement of a copper atom next to a $V_{\mathrm{Cu}}$ toward the vacant site such that it becomes coordinated to four oxygen atoms (i.e., two of which were previously coordinated to the displaced copper atom).

\section{Electronic structure of native defects}

As reported above, the DFT-calculated band gap of $\mathrm{Cu}_{2} \mathrm{O}$ $(0.46 \mathrm{eV})$ is notably smaller than the experimental value $(2.17 \mathrm{eV})$. Post-DFT corrections (e.g., with DFT $+U$ or with the $G W$ approximation) applied to DFT-GGA results would typically give rise to a larger band gap ${ }^{14,48-52}$ that is closer to the experimentally observed one. In this work, the corrections applied to the formation energy is the $\Delta v$ correction based on core levels, as well as the $\mathrm{Cu}_{2} \mathrm{O}$ band-gap correction due to Raebiger et al. ${ }^{14}$ In Ref. 14, the band gap of $\mathrm{Cu}_{2} \mathrm{O}$ was corrected by applying an effective Hubbard $U=6 \mathrm{eV}$ on the $\mathrm{Cu} 3 d$ states within the GGA $+U$ approach. This consequently pushes the VBM down by $0.32 \mathrm{eV}$, with the conduction-band maximum (CBM) arbitrarily extrapolated to match the experimental band gap of $2.1 \mathrm{eV}$. For our work, using the same band-gap correction value, we rigidly shift the VBM to lower energies by $0.32 \mathrm{eV}$. Taking these corrections into account, we find that our calculated relative formation energies of the native defects are qualitatively in good agreement with other reported theoretical values (which will be explained and discussed in a later section of this work).

For stoichiometric bulk $\mathrm{Cu}_{2} \mathrm{O}$, the calculated projected density of states (PDOS) in Fig. 2(a) shows that the VBM states (i.e., slightly below $E_{F}$ to $-5 \mathrm{eV}$ ) are predominantly $\mathrm{Cu} 3 d$ in character, with some $\mathrm{Cu} 3 d-\mathrm{O} 2 p$ hybridization at $0-2 \mathrm{eV}$. For states lying much lower in the valence band (from -5 to $-7.5 \mathrm{eV}$ ), hybridization of the $\mathrm{Cu} 3 d$ and $\mathrm{O} 2 p$ states can be seen as well with a larger component of $\mathrm{O} 2 p$ derived states. The lowest energy CBM states are of largely delocalized $\mathrm{Cu} 4 s$ character. This is inline with previous theoretical studies. ${ }^{14,48,49,53}$ We then examine the electronic structure of the various defects considered. To help identify the possible charge states for a given defect as well as to aid discussion, we summarized the defect-induced Kohn-Sham energy levels for the neutral state (in a 48-atom supercell) as a schematic energy diagram in Fig. 3.

Turning first to the interstitial and antisite defects, both $\mathrm{Cu}_{i \text { (oct) }}$ and $\mathrm{Cu}_{i \text { (tet) }}$ introduces a singlet $s$-like band close to the middle of the band gap, occupied with one electron and is hybridized with the CBM. This half-occupied defect band makes the copper interstitials amphoteric, i.e., having the ability to act both as a donor (allowing a +1 charge state) and an acceptor (i.e., affording a -1 charge state). The $\mathrm{Cu}_{i} 3 d$ states are deep in the valence band (from -1 to $-5 \mathrm{eV}$ ) and are largely delocalized. The $\mathrm{Cu}$ antisite $\mathrm{Cu}_{\mathrm{O}}$, on the other hand, induces defect states both deep in the valence band and a singly occupied triplet state in the conduction band. Due to the band filling of DFT, this electron is artificially placed at the CBM. Knowing that the GGA band gap is underestimated, it is possible that a more accurate description of the band structure may find that this defect level drops into the band gap, leading to the possibility of being negatively charged.

Both $\mathrm{O}_{i(\text { oct })}$ and $\mathrm{O}_{i(\text { tet })}$ exhibit rather similar behavior to each other. These two native defects introduce two localized states: one unoccupied singlet state in the band gap and an- 

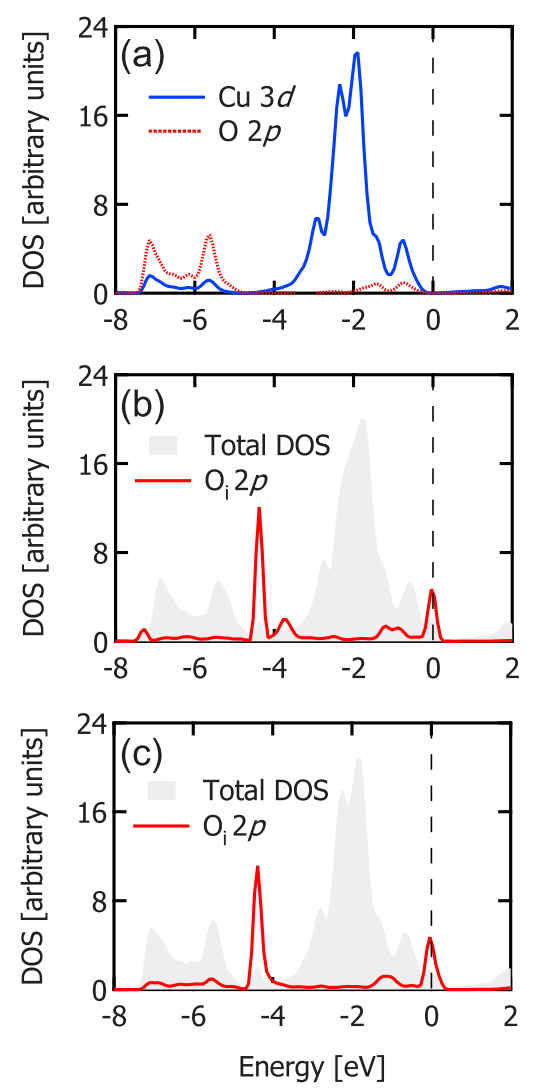

FIG. 2. (Color online) Spin-restricted projected density of states

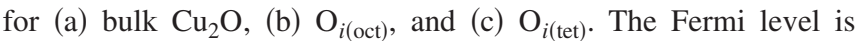
indicated by the vertical dashed line at $0 \mathrm{eV}$. High density of states at the Fermi level is seen for both oxygen defects, suggesting possible ferromagnetic behavior (see text for discussion).

other fully occupied doubly-degenerate state close to the top of the valence band. This defect thus acts as an acceptor which affords charge states of -1 and -2 . In addition, a deep localized state (at $\sim-4.5 \mathrm{eV}$ ) is also induced by these oxygen interstitial defects. Unlike the interstitials, the $\mathrm{O}$ antisite, $\mathrm{O}_{\mathrm{Cu}}$, introduces a hole in a doubly-degenerate state at the VBM, thus acting as a single acceptor (i.e., affords a -1 charge state).

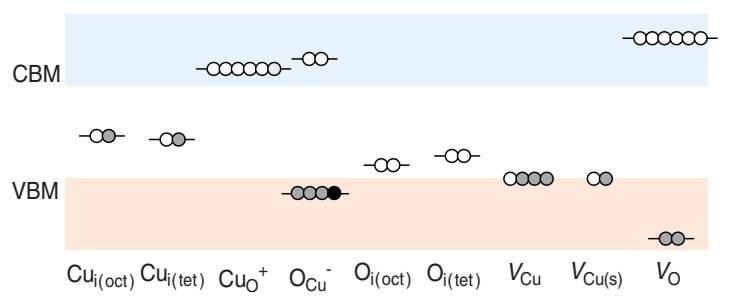

FIG. 3. (Color online) Schematic representation of the defectinduced energy levels in a 48-atom $\mathrm{Cu}_{2} \mathrm{O}$ supercell for the neutral charge state. The blue-shaded (darker) area represents the conduction band and the pink-shaded (lighter) area the valence band. Filled and open circles denote electrons and holes, respectively. The $\mathrm{Cu}$ antisite, $\mathrm{Cu}_{\mathrm{O}}$, is stable only in the +1 charge state, with the removed electron shown in a pale shade in the conduction band. The $\mathrm{O}$ antisite, $\mathrm{O}_{\mathrm{Cu}}$, is also stable only in the -1 charge state, with the added electron shown in a darker shade in the valence band.

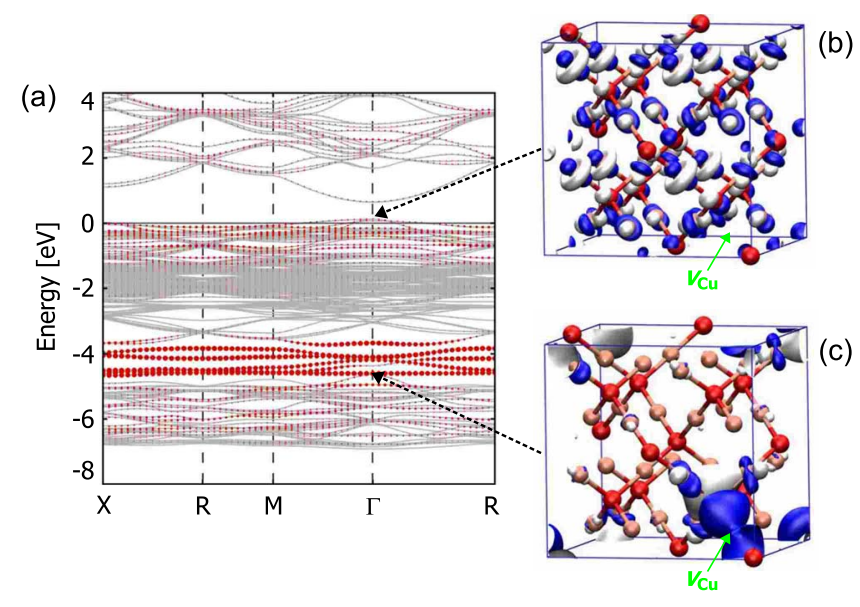

FIG. 4. (Color online) The band structure (with band character analysis) of $\mathrm{Cu}_{2} \mathrm{O}: V_{\mathrm{Cu}}$ is plotted in (a), with the solid lines representing the bands of the defect system and dots showing the weight (by dot size) of the contribution, by the nearest-neighbor oxygen atoms with unsaturated dangling bonds around the vacancy $\left(\mathrm{O}_{\mathrm{d}} 2 p\right)$, to the full band structure; (b) the wave function of the partially filled band at the $\Gamma$ point, showing a delocalized nature of the hole state generated by $V_{\mathrm{Cu}}$; (c) likewise for the deep localized defect state at $4 \mathrm{eV}$ deep in the valence band. For (b) and (c), copper atoms are shown as lighter spheres, and oxygen atoms as the darker spheres.

Creation of a copper vacancy, $V_{\mathrm{Cu}}$, in $\mathrm{Cu}_{2} \mathrm{O}$ introduces two equivalent oxygen atoms $\left(\mathrm{O}_{\mathrm{d}}\right)$ with unsaturated dangling bonds around the vacancy. $\mathrm{O}_{\mathrm{d}} 2 p$ states are found to dominate the electronic states around -4 to $-5 \mathrm{eV}$ from $E_{F}$, with a smaller contribution at around -1.5 to $0 \mathrm{eV}$. To understand the contribution of these states to the full band structure, we project the contributions of the $\mathrm{O}_{\mathrm{d}} 2 p$ states over the full band structure over a wider energy range in Fig. 4(a). The bands around -4 to $-5 \mathrm{eV}$ (dotted) are flat, distinctive of a localized defect state deep in the valence band. $V_{\mathrm{Cu}}$ generates a hole in a doubly-degenerate band at the VBM (indicated in Fig. 3), making it a single acceptor (i.e., allowing -1 charge state). To investigate the character of this hole state, we plot the wave functions of this partially occupied band at the $\Gamma$ point [see Fig. 4(b)] and find that it is largely delocalized. In contrast, the state deep in the valence band [at the $\Gamma$ point and $4 \mathrm{eV}$, see Fig. 4(c)] is largely localized around the vacancy. This implies that the hole produced by $V_{\mathrm{Cu}}$ "floats up" to the top of VBM and occupies a delocalized valence-band-like perturbed-host state (PHS). ${ }^{14,54}$ Unlike $V_{\mathrm{Cu}}$, the split-vacancy complex, $V_{\mathrm{Cu}(\mathrm{s})}$ induces a halffilled singlet state at the VBM, having the hybrid characteristics of both $V_{\mathrm{Cu}}$ and $\mathrm{Cu}_{i}$, acting as a single acceptor (i.e., allowing -1 charge state).

On the other hand, creating an oxygen vacancy, $V_{\mathrm{O}}$, explicitly forms four equivalent copper atoms $\left(\mathrm{Cu}_{\mathrm{d}}\right)$ with unsaturated dangling bonds around the vacancy. $\mathrm{Cu}_{\mathrm{d}} 3 d$ states are largely delocalized over the energy range of -1 to $-5 \mathrm{eV}$ from $E_{F}$, producing no localized defect-induced states in either the valence band nor the band gap. In contrast to the copper vacancy $V_{\mathrm{O}}$ introduces a fully occupied singlet state deep in the valence band and an empty triplet state inside the conduction band. This renders the oxygen vacancy 
incapable of acting as an acceptor; thus this defect is considered charge neutral and is not able to compensate any holes produced.

\section{Atomic structure of native defects}

The optimized atomic geometry of the native defects is shown in Fig. 1. For comparison, we first note that the calculated bond distance between a $\mathrm{Cu}$ atom and its nearest $\mathrm{O}$ atom, $d_{\mathrm{Cu}-\mathrm{O}}$, in bulk $\mathrm{Cu}_{2} \mathrm{O}$ is $1.87 \AA$, and the calculated bond distance between the two nearest $\mathrm{Cu}$ atoms, $d_{\mathrm{Cu}-\mathrm{Cu}}$, in bulk $\mathrm{Cu}$ is $2.58 \AA .{ }^{13,36}$ For $\mathrm{Cu}_{i \text { (oct) }}$ in the neutral charge state, we find that the bond distance of a $\mathrm{Cu}-\mathrm{O}$ atom pair in the host lattice surrounding the defect, $d_{\mathrm{Cu}-\mathrm{O}}$, undergoes a maximum bond stretch of $3.5 \%$ (resulting in a value of $1.94 \AA$ ) and a resulting bond distance of $2.46 \AA$ between the interstitial $\mathrm{Cu}$ and its neighboring host $\mathrm{Cu}$ atoms, $d_{\mathrm{Cu}_{i}-\mathrm{Cu}}$. The latter distance is $4.5 \%$ less than that of $d_{\mathrm{Cu}-\mathrm{Cu}}$. For the -1 charge state, the corresponding changes for $d_{\mathrm{Cu}-\mathrm{O}}$ and $d_{\mathrm{Cu}_{i}-\mathrm{Cu}}$ are $3.7 \%$ stretch and a bond length of $2.45 \AA$, respectively, while those for the +1 charge state are $3.7 \%$ for $d_{\mathrm{Cu}-\mathrm{O}}$, with a distance of $2.47 \AA$ for $d_{\mathrm{Cu}_{i}-\mathrm{Cu}}$. For $\mathrm{Cu}_{i(\text { tet })}$, the changes in both $d_{\mathrm{Cu}-\mathrm{O}}$ and $d_{\mathrm{Cu}_{i}-\mathrm{Cu}}$ exhibit a similar trend to that of $\mathrm{Cu}_{i \text { (oct) }}$ where, in the neutral charge state, a larger maximum bond stretch of $d_{\mathrm{Cu}-\mathrm{O}}(7.2 \%)$ and a similar value for $d_{\mathrm{Cu}_{i}-\mathrm{Cu}}$ $=2.44 \AA$ is found. This is, in part, a consequence of a smaller volume experienced by the interstitial $\mathrm{Cu}$ atom in the tetrahedral site, thus a larger distortion to the host lattice.

For the oxygen interstitials in the neutral charge states, in the case of $\mathrm{O}_{i \text { (oct) }}$, the bond distance between the interstitial oxygen and the nearest-neighbor $\mathrm{Cu}$ atoms surrounding the defect $d_{\mathrm{Cu}-\mathrm{O}_{i}}$ has an average value of $2.18 \AA$, which is $16.8 \%$ larger than that found for $d_{\mathrm{Cu}-\mathrm{O}}$ of the bulk host lattice. A much smaller value of $1.98 \AA$ is found in $\mathrm{O}_{i \text { (tet) }}$, i.e., a corresponding deviation of $6.1 \%$. Likewise, this difference can be explained by the fact that the octahedral interstitial site has a larger volume than that of the tetrahedral interstitial site, hence enforcing a more compact $d_{\mathrm{Cu}-\mathrm{O}}$ in $\mathrm{O}_{i(\text { tet })}$. The $\mathrm{Cu}-\mathrm{O}$ atom-pair distance, $d_{\mathrm{Cu}-\mathrm{O}}$, in the host lattice surround the oxygen defect is on average $1.93 \AA$, resulting in $\sim 3 \%$ stretch. For $\mathrm{O}_{i \text { (oct) }}$ in the -1 and -2 charge states, the $d_{\mathrm{Cu}-\mathrm{O}}$ bond distance remains relatively constant, with a value of 2.18 and $2.19 \AA$, respectively. The changes in $d_{\mathrm{Cu}-\mathrm{O}}$ for $\mathrm{O}_{i \text { (tet) }}$ are also negligible, having a value of $2.00 \AA$ for both the -1 and -2 charge states.

With regards to the copper antisite, $\mathrm{Cu}_{\mathrm{O}}$, we consider the nearest bond distance between $\mathrm{Cu}_{\mathrm{O}}$ and $\mathrm{Cu}$ from the host lattice $d_{\mathrm{Cu}_{\mathrm{O}}-\mathrm{Cu}}$, and the nearest $\mathrm{Cu}-\mathrm{O}$ bond in the coordination sphere of the host lattice, $d_{\mathrm{Cu}-\mathrm{O}}$. For the +1 charge state, the values of $d_{\mathrm{Cu}_{\mathrm{O}}-\mathrm{Cu}}$ and $d_{\mathrm{Cu}-\mathrm{O}}$ are found to be 2.21 and $1.87 \AA$, respectively. As for the oxygen antisite, $\mathrm{O}_{\mathrm{Cu}}$, the bond distance between the antisite defect and the nearest $\mathrm{O}$ atom, $d_{\mathrm{O}_{\mathrm{Cu}}{ }^{-} \mathrm{O}}$, and the nearest $\mathrm{Cu}-\mathrm{O}$ bond in the coordination sphere of the host lattice, $d_{\mathrm{Cu}-\mathrm{O}}$, is considered. In its -1 charge state, we obtain $d_{\mathrm{Cu}-\mathrm{O}}$ to be $1.85 \AA$, i.e., a small contraction of $1.1 \%$ from the bulk value and $d_{\mathrm{O}_{\mathrm{Cu}} \mathrm{O}}$ is $1.91 \AA$. In comparison to the calculated bond length of the oxygen molecule $(1.22 \AA),{ }^{13} d_{\mathrm{O}_{\mathrm{Cu}^{-} \mathrm{O}}}$ is $57 \%$ longer.
Turning to the vacancies, we begin with the simple copper vacancy, $V_{\mathrm{Cu}}$. We define $d_{\mathrm{O}_{\mathrm{d}}-\mathrm{O}_{\mathrm{d}}}$ as the distance between the two unsaturated $\mathrm{O}\left(\mathrm{O}_{\mathrm{d}}\right)$ atoms around the copper vacancy and similarly, $d_{\mathrm{Cu}-\mathrm{O}}$ as the distance between the nearest $\mathrm{Cu}-\mathrm{O}$ atom pair in the host lattice. For the neutral charge state, $d_{\mathrm{O}_{\mathrm{d}} \mathrm{O} \mathrm{d}}$ is found to be $3.52 \AA$ which is $5.8 \%$ shorter than the nearest $\mathrm{O}-\mathrm{O}$ bond distance in bulk $\mathrm{Cu}_{2} \mathrm{O}(3.74 \AA) . d_{\mathrm{Cu}-\mathrm{O}}$ is found to be $1.84 \AA$, i.e., a contraction of $1.4 \%$ from ideal. In its -1 charge state, the corresponding values are 3.49 and $1.85 \AA$ for $d_{\mathrm{O}_{\mathrm{d}} \mathrm{O}_{\mathrm{d}}}$ and $d_{\mathrm{Cu}-\mathrm{O}}$, respectively. Hence adding an additional negative charge to this defect draws the two $\mathrm{O}_{\mathrm{d}}$ atoms closer and thus lengthens the nearest host $\mathrm{Cu}-\mathrm{O}$ bond distances slightly. For the neutral copper split-vacancy complex, $V_{\mathrm{Cu}(\mathrm{s})}$, the distance between the inserted copper atom [see Fig. 1(1)] and the nearest host $\mathrm{O}$ atoms is found to be $2.16 \AA$ while that between the nearest host $\mathrm{Cu}-\mathrm{O}$ atom pair on average is $1.88 \AA$. In its -1 charge state, the corresponding values remain fairly constant.

The last native defect considered is the oxygen vacancy which exists only in the neutral charge state. We consider the distance between the four unsaturated $\mathrm{Cu}$ atoms $\left(\mathrm{Cu}_{\mathrm{d}}\right)$ due to the vacancy [shown in Fig. 1(h) as the four-atom tetrahedron cluster) as $d_{\mathrm{Cu}_{\mathrm{d}}-\mathrm{Cu}_{\mathrm{d}}}$. This is found to be $2.52 \AA$ which is $2.3 \%$ shorter than the $\mathrm{Cu}-\mathrm{Cu}$ distance found in bulk $\mathrm{Cu}(2.58 \AA)$. The distance between $\mathrm{Cu}_{\mathrm{d}}$ and its neighboring $\mathrm{O}$ atom is $2.01 \AA$, and that between the nearest $\mathrm{Cu}-\mathrm{O}$ atom pair in the host lattice $d_{\mathrm{Cu}-\mathrm{O}}$ is $1.90 \AA$. To investigate if such clustering of $\mathrm{Cu}$ atoms is favorable in $\mathrm{Cu}_{2} \mathrm{O}$ (upon the formation of oxygen vacancy defects), we perform two calculations in a 144-atom supercell, containing $2 V_{\mathrm{O}}$ defects: (1) A nearneighbor (NN) configuration: with two $V_{\mathrm{O}}$ placed adjacent to each other, such that the two tetrahedron $\mathrm{Cu}_{4}$ cluster is joined by one apex, and (2) a far-neighbor (FN) configuration: with two $V_{\mathrm{O}}$ maximally separated from each other $(\sim 9 \AA$ apart $)$. We find that the total-energy difference between the NN and FN configurations is $13.5 \mathrm{meV}$ per oxygen vacancy, with the FN configuration being more favorable. This serves as an indication that perhaps clustering of oxygen vacancies may not be favored while noting that such small energy differences could well be overcome at high growth temperatures. Similar to the single $V_{\mathrm{O}}$, both configurations are do not induce defect states in the gap.

\section{Formation energy and stability of native bulk defects}

To ascertain the error incurred from the supercell effect, we initially perform convergence tests for the formation energy of the copper vacancy (the lowest energy defect, as described below) for the neutral charge state using various supercells of increasing size. The results (under copper-rich conditions) are reported in Table I, also presenting the formation energies of $V_{\mathrm{Cu}(\mathrm{s})}, \mathrm{O}_{i \text { (oct) }}$, and $\mathrm{O}_{i \text { (tet) }}$ in the 48-atom supercell. For comparison, we have included reported values of the formation energies of these defects from Refs. 7 and 14. In Table I, it can be seen that, increasing from the 48atom to the 96-atom supercell, the defect formation energy of the neutral charge state decreases from 0.47 to $0.42 \mathrm{eV}$ (i.e., by $0.05 \mathrm{eV}$ ), and increasing further from the 96-atom to the 144-atom supercell, it lowers by yet another $0.08 \mathrm{eV}$ to a value of $0.34 \mathrm{eV}$. This value again decreases marginally to 
TABLE I. Defect formation energies (in $\mathrm{eV}$ ) of $V_{\mathrm{Cu}}, V_{\mathrm{Cu}(\mathrm{s})}$, $\mathrm{O}_{i(\text { tet })}$, and $\mathrm{O}_{i(\text { oct })}$ in the neutral charge state under copper-rich conditions. The size of $\mathrm{Cu}_{2} \mathrm{O}$ supercells are shown in parenthesis, e.g., (48) represents a 48-atom supercell. PAW-DFT(PBE) denotes the projector-augmented-wave method with the PBE approximation to the DFT exchange-correlation functional.

\begin{tabular}{lllll}
\hline \hline & $V_{\mathrm{Cu}}$ & $V_{\mathrm{Cu}(\mathrm{s})}$ & $\mathrm{O}_{i(\mathrm{tet})}$ & $\mathrm{O}_{i(\mathrm{oct})}$ \\
\hline Present work (48) & 0.47 & 0.78 & 1.47 & 1.90 \\
Present work (96) & 0.42 & & & \\
Present work (144) & 0.34 & & & \\
Present work (162) & 0.28 & & & \\
Raebiger et al. $(96)^{\mathrm{a}}$ & 0.7 & 1.0 & 1.3 & 1.8 \\
Nolan et al. $(48)^{\mathrm{b}}$ & 0.41 & & & \\
Nolan et al. $(162)^{\mathrm{b}}$ & 0.34 & & & \\
\hline
\end{tabular}

${ }^{\text {aRef. 14: PAW-DFT(PBE) }}$

${ }^{b}$ Ref. 7: PAW-DFT(PBE).

$0.28 \mathrm{eV}$ when increased to the 162-atom supercell. This clearly shows that, in principle, a rather large supercell is required to achieve absolute convergence. However, by plotting the calculated formation energies for all considered defects as a function of the Fermi energy (in Fig. 5) and comparing that to the recent work of Raebiger et al. (see Fig. 3 in Ref. 14), we find that the qualitative trend regarding the relative stability of the various defects is already captured when using the 48-atom supercell. Also from Table I, it is noted that reported values in this work appear to be lower (by 0.22 $\mathrm{eV}$ ) than that found by Raebiger et al. ${ }^{14}$ which uses the DFTGGA approach including additional corrections such as taking the image charge effect due to the supercell approach and (a)

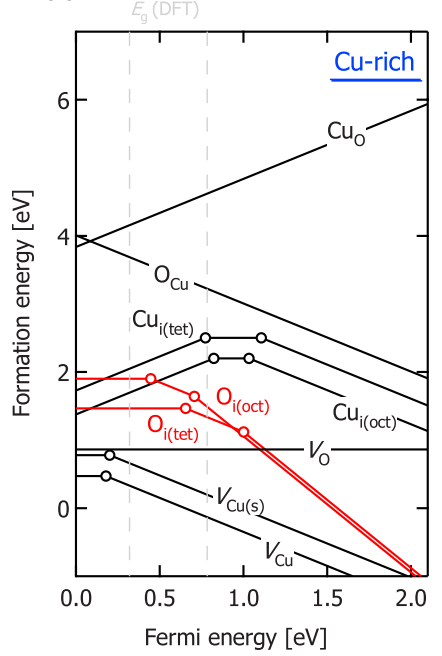

(b)

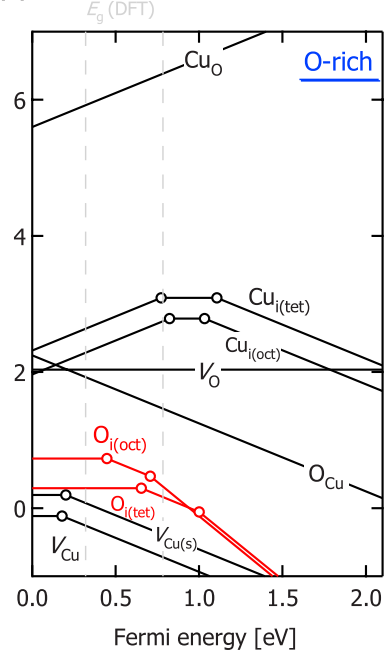

FIG. 5. (Color online) Defect formation energies for native defects in a 48-atom $\mathrm{Cu}_{2} \mathrm{O}$ supercell as a function of the Fermi energy under (a) copper-rich and (b) oxygen-rich conditions. Following Ref. 14, the VBM is adjusted by pushing down the DFT-derived VBM by $0.32 \mathrm{eV}$ while extending the CBM to match the experimental band gap of $2.17 \mathrm{eV} . \Delta E_{F}=0$ now corresponds to the VBM after this adjustment. The dashed vertical gray lines define the otherwise smaller DFT-calculated band gap. band-filling effect (for shallow defects). We tentatively attribute our differences to these additional corrections. Nevertheless, our value for the formation energy of $V_{\mathrm{Cu}}$ is in good agreement with that reported by Nolan et al.,$^{7}$ using conventional DFT-GGA, which is assuring. Overall, our calculated relative defect formation energy for the two copper vacancies and two oxygen interstitials are comparable with other theoretical works ${ }^{7,14}$ and will not change any qualitative trends identified in this work.

Having established the justifications (as well as limitations) of our current study, we present the defect formation energies of the various considered native defects as a function of $\Delta E_{F}$ under copper-rich and oxygen-rich conditions in Figs. 5(a) and 5(b), respectively. For clarity, we have not plotted the full straight line for each charged state of each defect but show only the section where that charge state is lower in energy than all other charge states. Therefore, the change in slope of the lines represents a change in the charge state of the defect [cf. Eq. (1)]. We note that the relative difference in the formation energies of the native defects between the oxygen-rich and copper-rich conditions, $\Delta E_{\mathrm{OR}-\mathrm{CR}}$, is expressed as

$$
\Delta E_{\mathrm{OR}-\mathrm{CR}}=\left(\frac{\Delta N_{\mathrm{Cu}}}{2}-\Delta N_{\mathrm{O}}\right) \Delta H_{\mathrm{Cu}_{2} \mathrm{O}}^{f},
$$

where $\Delta N_{\mathrm{Cu}}$ and $\Delta N_{\mathrm{O}}$ are the difference in the number of $\mathrm{Cu}$ and $\mathrm{O}$ atoms between the defect-containing supercell and the ideal reference supercell. $\Delta H_{\mathrm{Cu}_{2} \mathrm{O}}^{f}$ is the heat of formation of $\mathrm{Cu}_{2} \mathrm{O}$ which is defined as $\Delta H_{\mathrm{Cu}_{2} \mathrm{O}}^{f}=E_{\mathrm{Cu}_{2} \mathrm{O}}^{\mathrm{tot}}-E_{\mathrm{Cu} \text {,bulk }}^{\text {tot }}-\frac{1}{2} E_{\mathrm{O}_{2}}^{\text {tot }}$, where $E_{\mathrm{Cu}_{2} \mathrm{O}}^{\mathrm{tot}}, E_{\mathrm{Cu} \text {,bulk }}^{\mathrm{tot}}$, and $E_{\mathrm{O}_{2}}^{\mathrm{tot}}$ are the total energies of bulk $\mathrm{Cu}_{2} \mathrm{O}$, bulk $\mathrm{Cu}$, and the $\mathrm{O}_{2}$ gas-phase molecule, respectively. $\Delta H_{\mathrm{Cu}_{2} \mathrm{O}}^{f}$ is calculated to be $-1.24 \mathrm{eV}$ per formula unit. Using the copper antisite as an example, $\Delta N_{\mathrm{Cu}}=1$ and $\Delta N_{\mathrm{O}}=-1$, yielding $\Delta E_{\mathrm{OR}-\mathrm{CR}}=\frac{3}{2} \Delta H_{\mathrm{Cu}_{2} \mathrm{O}}^{f}$. Thus, $\Delta E_{\mathrm{OR}-\mathrm{CR}}$ for the copper vacancy and copper interstitials is $-\frac{1}{2} \Delta H_{\mathrm{Cu}_{2} \mathrm{O}}^{f}$ and $\frac{1}{2} \Delta H_{\mathrm{Cu}_{2} \mathrm{O}}^{f}$, respectively, and that for the oxygen vacancy and oxygen interstitials is $\Delta H_{\mathrm{Cu}_{2} \mathrm{O}}^{f}$ and $-\Delta H_{\mathrm{Cu}_{2} \mathrm{O}}^{f}$, respectively. For the oxygen antisite, $\Delta E_{\mathrm{OR}-\mathrm{CR}}=-\frac{3}{2} \Delta H_{\mathrm{Cu}_{2} \mathrm{O}}^{f^{2}}$. We also report the electronic transition levels for the various considered native defects in $\mathrm{Cu}_{2} \mathrm{O}$ in Table II, as defined by Eq. (3).

Under both copper- and oxygen-rich growth conditions (see Fig. 5), the simple copper vacancy has the lowest formation energy. Even under copper-rich conditions, its formation energy for the neutral charge state has a value of $0.47 \mathrm{eV}$ while the next lowest energy defect is the copper splitvacancy complex, being less favorable by $0.31 \mathrm{eV}$ for its corresponding neutral charge state. From Table II, the calculated $\Delta \epsilon(0 /-1)$ for $V_{\mathrm{Cu}}$ and $V_{\mathrm{Cu}(\mathrm{s})}$ are 0.18 and $0.20 \mathrm{eV}$, respectively. The corresponding values reported by Raebiger et $a{ }^{14}$ is slightly higher, being 0.28 and $0.30 \mathrm{eV}$. These theoretical values are found to be comparatively lower than the experimental value of $0.45 \mathrm{eV}$, determined by deep-level transient spectroscopy. ${ }^{55}$ In this work, Paul et al. ${ }^{55}$ also identified another lower trap level of $0.25 \mathrm{eV}$ and have tentatively assigned that as a $\mathrm{Cu}$ divacancy. Referring to Table I, it is evident that the formation energy of $V_{\mathrm{Cu}}$ decreases with increasing supercell size. This is indicative of a repulsive 
TABLE II. Calculated transition levels, $\Delta \epsilon\left(q / q^{\prime}\right)$ [cf. Eq. (3)] (in $\mathrm{eV}$ ) for the various considered native defects in $\mathrm{Cu}_{2} \mathrm{O}$ (within a 48-atom supercell). No transition levels are designated for both $\mathrm{Cu}$ and $\mathrm{O}$ antisites and the $\mathrm{O}$ vacancy (see text for discussion). It is to be noted that the reference VBM $\epsilon_{\mathrm{VBM}}^{\text {bulk }}$ [see Eq. (1)] has been corrected, i.e., rigidly shifting to lower energies by $0.32 \mathrm{eV}$ (Ref. 14). PAW-DFT(PBE) denotes the projector-augmented-wave method with the PBE approximation to the DFT exchange-correlation functional.

\begin{tabular}{lccc}
\hline \hline & $\Delta \epsilon(+1 / 0)$ & $\Delta \epsilon(0 /-1)$ & $\Delta \epsilon(-1 /-2)$ \\
\hline$V_{\mathrm{Cu}}$ & & 0.18 & \\
$V_{\mathrm{Cu}(\mathrm{s})}$ & & 0.20 & \\
$\mathrm{Cu}_{i(\text { oct })}$ & 0.82 & 1.03 & \\
$\mathrm{Cu}_{i(\text { tet })}$ & 0.77 & 1.11 & 0.71 \\
$\mathrm{O}_{i(\text { oct })}$ & & 0.45 & 1.00 \\
$\mathrm{O}_{i(\text { tet })}$ & & 0.65 & \\
$V_{\mathrm{Cu}}{ }^{\mathrm{a}}$ & & 0.28 & \\
$V_{\mathrm{Cu}(\mathrm{s})}{ }^{\mathrm{a}}$ & & 0.30 & \\
$\mathrm{Cu}_{i(\text { oct })}{ }^{\mathrm{a}}$ & 0.98 & 1.47 & \\
$\mathrm{Cu}_{i(\text { tet })}{ }^{\mathrm{a}}$ & 1.00 & 1.50 & 1.35 \\
$\mathrm{O}_{i(\text { oct })}{ }^{\mathrm{a}}$ & & 0.66 & 1.50 \\
$\mathrm{O}_{i(\text { (tet })}{ }^{\mathrm{a}}$ & & 0.78 & \\
$V_{\mathrm{Cu}}{ }^{\mathrm{b}}$ & & $0.45,0.25$ \\
\hline \hline
\end{tabular}

aef. 14: PAW-DFT(PBE).

${ }^{b}$ Ref. 55: Deep-level transient spectroscopy. $\Delta \epsilon(0 /-1)=0.25 \mathrm{eV}$ is tentatively assigned to a $\mathrm{Cu}$ divacancy.

$V_{\mathrm{Cu}}-V_{\mathrm{Cu}}$ interaction and suggests that copper vacancies would not prefer to cluster, assuming they would form homogeneously. Interestingly, under oxygen-rich growth conditions, oxygen interstitial defects, in addition to the two above-mentioned copper vacancies, are also low-energy defects, having a formation energy of 0.29 and $0.73 \mathrm{eV}$ for $\mathrm{O}_{i \text { (tet) }}$ and $\mathrm{O}_{i(\text { oct })}$, respectively, in the neutral charge state. To examine if the oxygen interstitial defects prefer to cluster, using $\mathrm{O}_{i \text { (oct) }}$ as an example, we find also that the formation energy of this defect (in the neutral charge state) in a 144atom supercell is $21.1 \mathrm{meV}$ more favorable than that in the 48-atom supercell, indicating a repulsive interaction between $\mathrm{O}_{i \text { (oct) }}$ defects. Similarly to the copper vacancy, the oxygen interstitials are unlikely to form cluster defect complexes.

In order to have prevalent $p$-type conductivity in this material, the concentration of defects that compensate any holes produced should be kept low, i.e., suppressing the formation of hole killers. ${ }^{14}$ In $\mathrm{Cu}_{2} \mathrm{O}$, such defect species are the copper interstitials $\left(\mathrm{Cu}_{i \text { (oct) }}\right.$ and $\left.\mathrm{Cu}_{i(\text { tet })}\right)$ and copper antisite $\left(\mathrm{Cu}_{\mathrm{O}}\right)$. Even though the oxygen vacancy has a rather low formation energy under copper-rich conditions, it is only stable in the neutral charge state, rendering it unable to compensate any holes produced. Similarly, copper interstitials have significantly higher formation energies under both growth conditions; hence their defect concentrations are negligible compared to hole producers such as $V_{\mathrm{Cu}}$. Finding a similar trend to Ref. 14, the formation energies of the copper vacancies and oxygen interstitials are lowered while that of the oxygen vacancy and copper interstitials are increased, when going
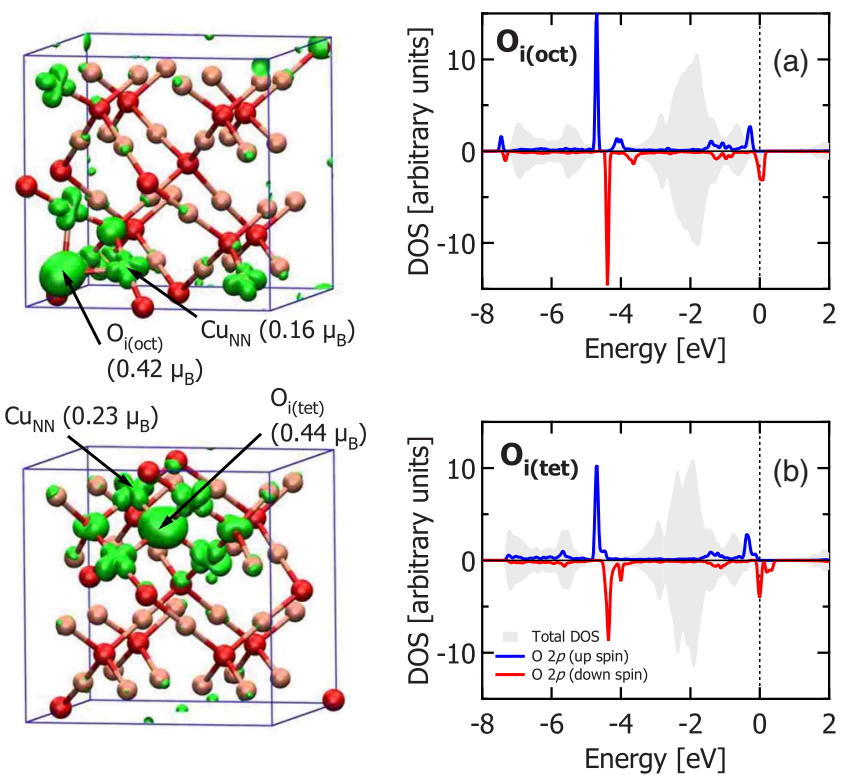

FIG. 6. (Color online) Oxygen-related defects: Spin densities (left panel) and spin-resolved projected density of states (right panel) are plotted for (a) $\mathrm{O}_{i(\mathrm{oct})}$ and (b) $\mathrm{O}_{i(\mathrm{tet})}$. The magnetic moments on the oxygen interstitial (labeled as $\mathrm{O}_{i(\text { oct })}$ and $\left.\mathrm{O}_{i(\text { tet })}\right)$ and its nearest-neighbor $\mathrm{Cu}$ atom $\left(\mathrm{Cu}_{\mathrm{NN}}\right)$ are reported in the parenthesis. Copper atoms are shown as lighter (orange) spheres, and oxygen atoms as the darker (red) spheres.

from the copper-rich conditions to oxygen-rich conditions. However, from Table II, we observe that the transition levels for both interstitial oxygen are much higher than that of the copper vacancies, thus rendering them harder to ionize than the copper vacancies. Their tendency to form under oxygenrich conditions will not contribute to the prevalent $p$-type conductivity in this material. From this analysis, it is now apparent why cuprous oxide is commonly known as a substoichiometric semiconductor. ${ }^{33,34}$

\section{Magnetic structure of low-energy defects}

Having identified the low-energy native defects under copper-rich and oxygen-rich conditions, namely, the single copper vacancy, copper split-vacancy complex and two oxygen interstitials, we now investigate their magnetic structure and properties. For each of these bulk defects, we first perform a spin-polarized calculation in a 48-atom supercell, relaxing all atoms in the cell. We find that $V_{\mathrm{Cu}}$ and $V_{\mathrm{Cu}(\mathrm{s})}$ are nonmagnetic for both the neutral and -1 charge state. Thus for the bulk material, despite having such low formation energies (i.e., should exist in high concentrations under growth conditions), these copper defects are not responsible for the experimentally observed magnetism. ${ }^{31,32}$

On the other hand, the oxygen interstitial defects $\mathrm{O}_{i \text { (oct) }}$ and $\mathrm{O}_{i(\text { tet })}$ are found to be magnetic. We first examine the magnetic properties for the neutral defects before proceeding to the charged defects. Similar total magnetic moments of $1.38 \mu_{B}$ and $1.36 \mu_{B}$ are found for the $\mathrm{O}_{i \text { (oct) }}$ and $\mathrm{O}_{i \text { (tet) }}$ neutral defect systems. The spin densities and spin-resolved PDOS for these two defects are shown in Fig. 6. We can clearly see that the spin densities of these two oxygen interstitial defects 
are localized largely on the interstitial oxygen with its nearest-neighbor copper atoms slightly spin polarized. For $\mathrm{O}_{i \text { (oct) }}[$ see Fig. 6(a)], the local spin moment on the interstitial oxygen is calculated to be $0.42 \mu_{B}$ while its six nearestneighboring copper atoms are polarized slightly by $0.16 \mu_{B}$ each, giving a ferromagnetic ordering, with a total magnetic spin moment of $1.38 \mu_{B}$. As for $\mathrm{O}_{i \text { (tet) }}$ [see Fig. 6(b)], the interstitial oxygen has a spin moment of $0.44 \mu_{B}$ with each of its four nearest-neighboring copper atoms having a spin moment of $0.23 \mu_{B}$ each. This results in a ferromagnetic ordering with a total spin moment of $1.36 \mu_{B}$. For both oxygen defects, other atoms in the supercell are spin polarized with not more than $0.02 \mu_{B}$. For $\mathrm{O}_{i(\mathrm{oct})}$ in the neutral state, the antiferromagnetic solution is found to be $17.6 \mathrm{meV}$ less stable than the ferromagnetic state and similarly, for $\mathrm{O}_{i(\text { tet })}$ in the neutral charge state, the antiferromagnetic state is also less favorable with respect to the ferromagnetic solution by about $10 \mathrm{meV}$. The spin-polarized formation energy of $\mathrm{O}_{i \text { (oct) }}$ in the neutral charge state is found to be $127.3 \mathrm{meV}$ more stable than the spin-restricted value. For $\mathrm{O}_{i \text { (tet) }}$ in the neutral charge state, this difference is smaller, i.e., $54.7 \mathrm{meV}$.

This spin ordering is, in part, reflected in the spinresolved PDOS in Figs. 6(a) and 6(b). Ferromagnetic spin ordering is favored when a large number of electrons can shift favorably to nonoccupied states over a small energy difference. Hence, an important quantity for this occurence is the density of states at the Fermi level, $N\left(E_{F}\right) .{ }^{56}$ The Stoner criterion for ferromagnetic ordering, $\alpha_{S} N\left(E_{F}\right)>1$, where $\alpha_{S}$ is the Stoner parameter, predicts whether the energy gained through the exchange interaction by aligning a ferromagnetic spin is larger than the energy required to reorder the band occupancy. ${ }^{56}$ Thus, assuming $\alpha_{S}$ remains comparatively constant for our system, the decisive factor in establishing a ferromagnetic ordering will be largely played by $N\left(E_{F}\right)$. For both oxygen interstitial defects, the spin-restricted PDOS [see Figs. 2(b) and 2(c)] clearly indicates a high $N\left(E_{F}\right)$, which in part fulfills the Stoner's criterion, resulting in a half-metallic character for the spin-resolved PDOS [Figs. 6(a) and 6(b)], with the spin-up states predominantly occupied. Characteristic sharp localized peaks deep in the VB (at -4 to $-5 \mathrm{eV}$ ) are seen for both defects, and are largely attributed to the bonding states between $\mathrm{O}_{i} 2 p$ and $\mathrm{Cu} 3 d$.

Turning to the charged defects, the total magnetic moments for both oxygen interstitial defects are reduced as compared to the neutral state. The total magnetic moment for $\mathrm{O}_{i \text { (oct) }}$ in the -1 charge state is calculated to be $0.74 \mu_{B}$ while that of the -2 charge state is quenched, i.e., reduced to zero. Similarly, for $\mathrm{O}_{i(\text { tet })}$, the corresponding value for the -1 charge state is $0.75 \mu_{B}$ while that of the -2 charge state, unlike $\mathrm{O}_{i \text { (oct) }}$, is not quenched but takes a smaller value of $0.33 \mu_{B}$. The difference in the spin-polarized and spinrestricted formation energies of these charged defects are smaller as compared to their corresponding neutral states. For $\mathrm{O}_{i \text { (oct) }}$ in the -1 charge state, it is $60.2 \mathrm{meV}$ more favorable in the spin-polarized form. Likewise, for $\mathrm{O}_{i(\mathrm{tet})}$, the corresponding value for the -1 charge state is $33.4 \mathrm{meV}$ and that for the -2 charge state is $7.0 \mathrm{meV}$.

Hence, summarizing the results obtained for the bulk native defects, one finds that, under both limits of growth conditions, copper vacancies are very favorable even under copper-rich conditions and is the main source of $p$-type conductivity in this material. The concentrations of these defects have been estimated to range between $2 \times 10^{18}$ and $2 \times 10^{20} \mathrm{~cm}^{-3} \cdot{ }^{14}$ However, these copper defects are calculated to be spin neutral (i.e., nonmagnetic) in both the neutral and -1 charge states, and therefore are not predicted within DFT to contribute to the magnetism observed experimentally. On the other hand, especially under oxygen-rich conditions, ferromagnetic oxygen-related bulk native defects, namely, the oxygen interstitials, have sufficiently low formation energy (i.e., concentrations estimated to be only about one to two orders of magnitude lower than that of $V_{\mathrm{Cu}}$ ) and are ferromagnetically ordered. This seems to suggest that one should expect measurable magnetism in bulk $\mathrm{Cu}_{2} \mathrm{O}$, particularly under oxygen-rich growth conditions. Nevertheless, when nanoparticles $\left(10-100 \mathrm{~nm}\right.$ in size) of $\mathrm{Cu}_{2} \mathrm{O}$ are considered, rather than simply considering bulk properties, what happens at the surface of these nanoparticles becomes important. Therefore, we now proceed to investigate how surface native defects and microfaceted (i.e., vicinal, high-index) oxide surfaces could possibly play a role in the magnetic properties of low-dimensional $\mathrm{Cu}_{2} \mathrm{O}$ nanostructures, especially in nanothin films and polycrystalline samples.

\section{Oxide surfaces: Thermodynamics and magnetism}

In our previous study, ${ }^{13}$ we investigated both stoichiometric and nonstoichiometric low-index oxide surfaces, namely, the (100), (110), and (111) surfaces of $\mathrm{Cu}_{2} \mathrm{O}$. We calculated the surface free energy for various surface terminations and presented these energies as a function of the oxygen chemical potential. We found that two surfaces, none of which are stoichiometric: the $\mathrm{Cu}_{2} \mathrm{O}(110): \mathrm{CuO}$ surface which is terminated with both $\mathrm{Cu}$ and $\mathrm{O}$ atoms, and the $\mathrm{Cu}_{2} \mathrm{O}(111)-\mathrm{Cu}_{\mathrm{CUS}}$ surface which contains a surface (coordinatively unsaturated) $\mathrm{Cu}_{\text {CUS }}$ vacancy, exhibit the lowest surface free energy for the considered range of oxygen chemical potential. The atomic geometry of $\mathrm{Cu}_{2} \mathrm{O}(110): \mathrm{CuO}$ and stoichiometric $\mathrm{Cu}_{2} \mathrm{O}(111)$ are shown again in Figs. 7(a) and 7(b), respectively.

In this work, we extend our study of oxide surfaces to include high-index or vicinal surfaces, namely, the (210) and (311) surfaces of $\mathrm{Cu}_{2} \mathrm{O}$. These so-called microfaceted oxide surfaces can be considered as a first step to understand the possible surface properties of polycrystalline $\mathrm{Cu}_{2} \mathrm{O}$. More commonly used to describe vicinal metal surfaces, ${ }^{57}$ vicinal $\mathrm{Cu}_{2} \mathrm{O}$ surfaces could also alternatively be described by a terrace $\times$ step notation whereby the terraces and steps are common low-index surface planes, in conjunction with the commonly used Miller indices in this work. Thus, $\mathrm{Cu}_{2} \mathrm{O}(210)$ can be written as $2(110) \times(100)$, and $\mathrm{Cu}_{2} \mathrm{O}(311)$ as $2(100) \times(111)$. This translates to $\mathrm{Cu}_{2} \mathrm{O}(210)$ having two repeating units of the low-index (110) surface on the terraces and one unit of (100) at the steps. Correspondingly, $\mathrm{Cu}_{2} \mathrm{O}(311)$ will have two units of (100) on the terrace and one unit of (111) at the steps. Adding on to this, the (110) surface is terminated with both $\mathrm{Cu}$ and $\mathrm{O}$, while the (100) surface is $\mathrm{O}$ terminated [previously identified as (110): $\mathrm{CuO}$ 

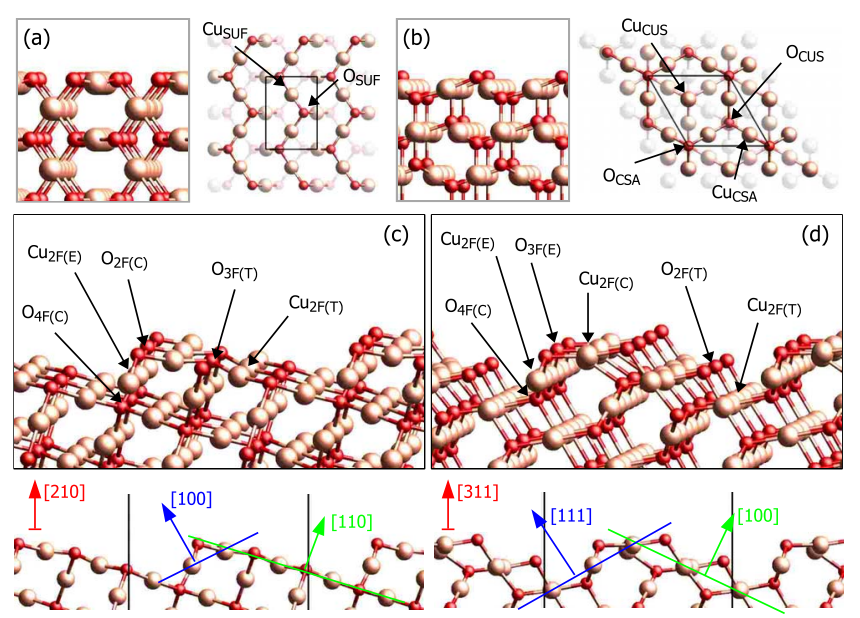

FIG. 7. (Color online) Surface structures of $\mathrm{Cu}_{2} \mathrm{O}$ : (a) and (b) show perspective side and top views of $\mathrm{Cu}_{2} \mathrm{O}(110): \mathrm{CuO}$ and $\mathrm{Cu}_{2} \mathrm{O}(111)$, respectively. (c) and (d) show the perspective side view of $\mathrm{Cu}_{2} \mathrm{O}(210)$ and $\mathrm{Cu}_{2} \mathrm{O}(311)$, respectively, with the corresponding low-index planar directions indicated in the lower panel. Copper atoms are depicted as large (orange) circles, and oxygen atoms as the smaller (red) circles. For the high-index (210) and (311) surfaces, the notation used for the surface atoms is as follows: $X_{n F(Y)}$ where $X$ is either $\mathrm{Cu}$ or $\mathrm{O}, n$ is the number of neighboring atoms $X$ is bonded to, and $Y$ denotes the geometric location of $X$, which could be a corner $(C)$, step $(E)$, or terrace $(T)$ site. For $\mathrm{Cu}_{2} \mathrm{O}(110): \mathrm{CuO}$ and $\mathrm{Cu}_{2} \mathrm{O}(111)$, the surface unit cells are shown in black lines while the repeating unit for $\mathrm{Cu}_{2} \mathrm{O}(210)$ and $\mathrm{Cu}_{2} \mathrm{O}(311)$ are shown between the vertical black lines in the lower panel.

and (100):O, respectively, in Ref. 36]. The (111) surface remains stoichiometric. The atomic geometries of these vicinal surfaces are depicted in Figs. 7(c) and 7(d), respectively, showing the corresponding low-index planar directions.

Upon relaxation, the atomic geometries of these surfaces reconstruct to give a more compact surface structure. To discuss changes in the $\mathrm{Cu}-\mathrm{O}$ bond lengths, the notation used for the surface atoms is as follows: $X_{n F(Y)}$, where $X$ is either $\mathrm{Cu}$ or $\mathrm{O}, n$ is the number of neighboring atoms $X$ is bonded to, and $Y$ denotes the geometric location of $X$, which could be a corner $(C)$, step $(E)$, or terrace $(T)$ site. The corner site is defined as the intersection site of a step and terrace plane. As an example, an oxygen atom fourfold coordinated to four neighboring $\mathrm{Cu}$ atoms at a corner site would represented by $\mathrm{O}_{4 F(C)}$. Table III reports the average optimized bond distances between surface $\mathrm{Cu}$ and $\mathrm{O}$ atoms in the $\mathrm{Cu}_{2} \mathrm{O}(210)$ and $\mathrm{Cu}_{2} \mathrm{O}(311)$ systems. In general, the $\mathrm{Cu}-\mathrm{O}$ distances are contracted to give a more compact surface structure with the exception of a few $\mathrm{Cu}-\mathrm{O}$ distances which are stretched relative to the bulk $\mathrm{Cu}-\mathrm{O}$ distance $(1.87 \AA)$.

Using the same approach of "ab initio atomistic thermodynamics" as described in Ref. 36, we calculate the surface Gibbs free energy of these two vicinal surfaces as a function of the chemical potential of oxygen and combine the results from our present and previous works in Fig. 8. Within this approach, the surface Gibbs free energy, $\gamma\left(p_{i}, T\right)$ is defined as
TABLE III. Optimized average surface $\mathrm{Cu}-\mathrm{O}$ bond distances, $d_{\mathrm{Cu}-\mathrm{O}}\left(\right.$ in $\AA$ ), for $\mathrm{Cu}_{2} \mathrm{O}(210)$ and $\mathrm{Cu}_{2} \mathrm{O}(311) . \Delta d$ (in \%) is the percentage difference in the bond distance to the ideal $d_{\mathrm{Cu}-\mathrm{O}}$ in $\mathrm{Cu}_{2} \mathrm{O}$ which is $1.87 \AA$ A. For explicit meaning of surface-atom notation, we refer the reader to the text and Figs. 7(c) and 7(d).

\begin{tabular}{llcr}
\hline \hline Surface & $\mathrm{Cu}-\mathrm{O}$ bond & $d_{\mathrm{Cu}-\mathrm{O}}$ & $\Delta d$ \\
\hline $\mathrm{Cu}_{2} \mathrm{O}(210)$ & $\mathrm{Cu}_{2 F(T)}-\mathrm{O}_{3 F(T)}$ & 1.84 & -1.6 \\
& $\mathrm{Cu}_{2 F(T)}-\mathrm{O}_{2 F(C)}$ & 1.78 & -4.8 \\
& $\mathrm{Cu}_{2 F(E)}-\mathrm{O}_{2 F(C)}$ & 1.78 & -4.8 \\
& $\mathrm{Cu}_{2 F(E)}-\mathrm{O}_{4 F(C)}$ & 1.90 & 1.6 \\
& $\mathrm{Cu}_{2 F(T)}-\mathrm{O}_{4 F(C)}$ & 1.88 & 0.5 \\
$\mathrm{Cu}_{2} \mathrm{O}(311)$ & $\mathrm{Cu}_{2 F(T)}-\mathrm{O}_{2 F(T)}$ & 1.77 & -5.3 \\
& $\mathrm{Cu}_{2 F(E)}-\mathrm{O}_{4 F(C)}$ & 1.89 & 1.1 \\
& $\mathrm{Cu}_{2 F(C)}-\mathrm{O}_{3 F(E)}$ & 1.84 & -1.6 \\
& $\mathrm{Cu}_{2 F(E)}-\mathrm{O}_{3 F(E)}$ & 1.83 & -2.1 \\
\hline \hline
\end{tabular}

$$
\gamma\left(p_{i}, T\right)=\frac{1}{A}\left[G-\sum_{i} N_{i} \mu_{i}\left(p_{i}, T\right)\right],
$$

where $G$ is the Gibbs free energy of the system with the surface area $A$ and $\mu_{i}\left(p_{i}, T\right)$ is the chemical potential of the various species $i$ present in the system, i.e., in this case $i=\mathrm{Cu}$ and $\mathrm{O} . N_{i}$ is the number of atoms of the $i$ th component in the system. For ease of comparison, we have only shown the most stable termination for the various lowindex surfaces, i.e., $\mathrm{Cu}_{2} \mathrm{O}(100): \mathrm{O}, \mathrm{Cu}_{2} \mathrm{O}(110): \mathrm{CuO}$, and $\mathrm{Cu}_{2} \mathrm{O}(111)-\mathrm{Cu}_{\mathrm{CUS}}$, from our previous work. ${ }^{36}$ To investigate if $\mathrm{Cu}$ vacancies at the surface of $\mathrm{Cu}_{2} \mathrm{O}(111)$ prefer to cluster, we also performed an additional calculation with a larger $(2 \times 2)$ surface supercell of $\mathrm{Cu}_{2} \mathrm{O}(111)$ and removed one $\mathrm{Cu}_{\mathrm{CUS}}$, i.e., having a quarter of the surface defect concentration of $\mathrm{Cu}_{2} \mathrm{O}(111)-\mathrm{Cu}_{\mathrm{CUS}}$. We find that, for the considered range of oxygen chemical potential, the surface energy calculated for this structure is much higher than that of just removing one $\mathrm{Cu}_{\mathrm{CuS}}$ from $\mathrm{Cu}_{2} \mathrm{O}(111)$ (see Fig. 8). This implies that clustering of $\mathrm{Cu}_{\mathrm{CUS}}$ vacancy is energetically favored, i.e., there is an attractive interaction between the surface vacancies.

Regarding surface stoichiometry of the consider oxide surfaces, we define $R_{\mathrm{Cu} / \mathrm{O}}$ to be the ratio of the number of $\mathrm{Cu}$ atoms to that of oxygen atoms in the surface structure. This implies that a stoichiometric surface will have $R_{\mathrm{Cu} / \mathrm{O}}=2$. Consequently, the oxide structures which have their surface free energies plotted in Fig. 8 are considered oxygen-rich terminated (i.e., having more $\mathrm{O}$ atoms than the stoichiometric surface hence $R_{\mathrm{Cu} / \mathrm{O}}<2$ ), thus showing a negative slope. This indicates that these surface terminations are more favorable in an oxygen-rich gaseous environment. Comparing the results in Fig. 8, it becomes apparent that the vicinal surfaces are not stable with respect to the previously identified low-energy surfaces-namely, $\mathrm{Cu}_{2} \mathrm{O}(110): \mathrm{CuO}$ and $\mathrm{Cu}_{2} \mathrm{O}(111)-\mathrm{Cu}_{\text {CUs }}$.

To investigate the (de)polarization effects at these oxide surfaces, we calculate the work function, $\Phi$, for selected surface structures, defined according to Eq. (7), 


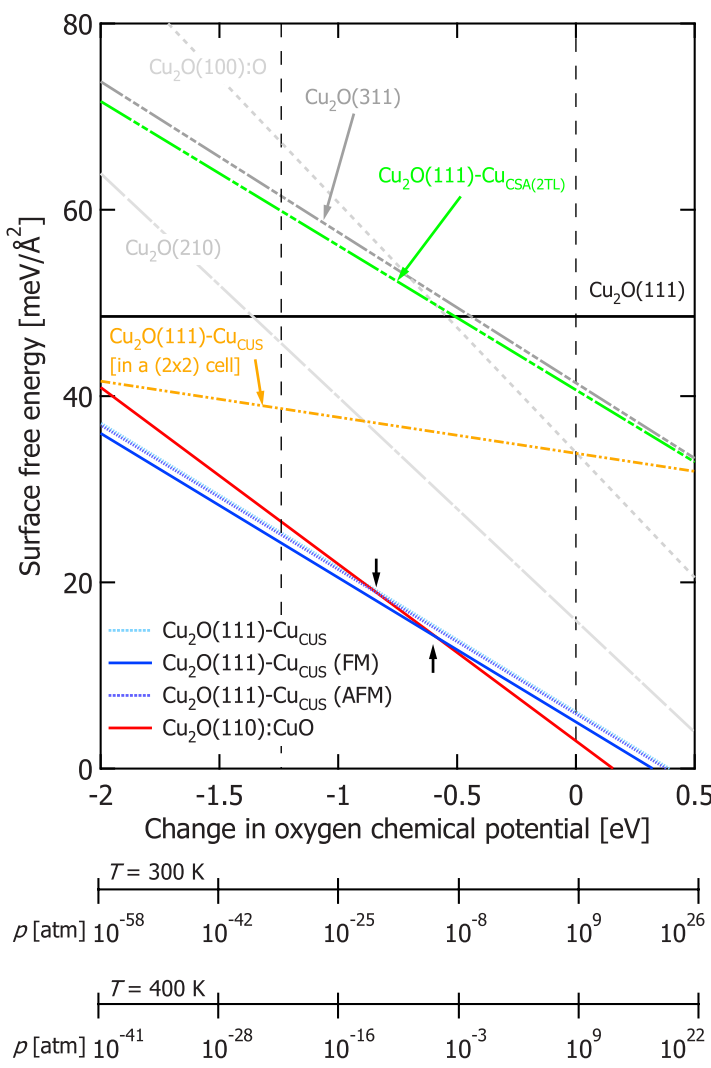

FIG. 8. (Color online) Calculated surface free energy of various considered $\mathrm{Cu}_{2} \mathrm{O}$ surfaces as a function of the change in oxygen chemical potential, $\Delta \mu_{\mathrm{O}}$, with the corresponding pressure bar lines at $T=300$ and $400 \mathrm{~K}$. Two competing low-energy structures $\left[\mathrm{Cu}_{2} \mathrm{O}(110): \mathrm{CuO}\right.$ and $\left.\mathrm{Cu}_{2} \mathrm{O}(111)-\mathrm{Cu}_{\mathrm{CUS}}\right]$ are found. Small vertical arrows indicate the crossover point between the stability of these two surfaces.

$$
\Phi=V_{\infty}-E_{F},
$$

where $V_{\infty}$ and $E_{F}$ are the electrostatic potential at infinity (i.e., in the middle of the vacuum region of the slab) and the Fermi energy, respectively. The calculated values are reported in Table IV.

The work function is a direct consequence of the electrostatic barrier induced by the dipole double layer at the surface. ${ }^{58}$ The presence of highly electronegative atoms, such as oxygen, at the surface (especially for $R_{\mathrm{Cu} / \mathrm{O}}<2$ ) increases the contribution of the dipole double layer to this electrostatic barrier, making it harder for an electron to leave the surface. This is reflected in the work functions of these substoichiometric surfaces which have values higher than that of the stoichiometric $\mathrm{Cu}_{2} \mathrm{O}(111)$ surface. Vicinal surfaces also tend to have a higher work function due to complex surface corrugations present at these surfaces.

We now begin to investigate the magnetic structure of these low-energy surfaces. Having identified that copper vacancies in the bulk material is nonmagnetic, it is indeed surprising to find that, by removing $\mathrm{Cu}_{\mathrm{CUS}}$ from the surface of $\mathrm{Cu}_{2} \mathrm{O}(111)$, results in an appreciable magnetic moment of $0.91 \mu_{B}$, noting that the defect-free stoichiometric $\mathrm{Cu}_{2} \mathrm{O}(111)$ is nonmagnetic. The spin-(un)restricted surface band struc-
TABLE IV. Work functions, $\Phi$, for selected surface structures. $R_{\mathrm{Cu} / \mathrm{O}}$ is the ratio of the number of $\mathrm{Cu}$ atoms to that of oxygen atoms in the surface structure. A stoichiometric surface will have $R_{\mathrm{Cu} / \mathrm{O}}=2$.

\begin{tabular}{lcc}
\hline \hline Surface structures & $R_{\mathrm{Cu} / \mathrm{O}}$ & $\begin{array}{c}\Phi \\
(\mathrm{eV})\end{array}$ \\
\hline $\mathrm{Cu}_{2} \mathrm{O}(111)-\mathrm{Cu}_{\mathrm{CUS}}$ & 1.80 & 5.36 \\
$\mathrm{Cu}_{2} \mathrm{O}(210)[2(110) \times(100)]$ & 1.82 & 6.32 \\
$\mathrm{Cu}_{2} \mathrm{O}(311)[2(100) \times(111)]$ & 1.83 & 5.97 \\
$\mathrm{Cu}_{2} \mathrm{O}(110): \mathrm{CuO}$ & 1.89 & 5.89 \\
$\mathrm{Cu}_{2} \mathrm{O}(111)$ & 2.00 & 4.58 \\
\hline \hline
\end{tabular}

tures and spin densities for $\mathrm{Cu}_{2} \mathrm{O}(111)-\mathrm{Cu}_{\text {CUS }}$ are plotted in Fig. 9 with the projected bulk states shaded in gray. To account for the magnetic moment observed at this surface, it is clear from the spin-restricted surface band structure in Fig. 9(a) that many highly localized surface states are found near the Fermi level, indicating a possible Stoner instability. Thus, taking spin polarization into account, the spin-up and spindown states are plotted in Figs. 9(b) and 9(c), respectively. The spin-up states near the Fermi level is fully occupied while the spin-down bands cut the Fermi level, indicating partial occupancy. This half-metallic behavior is similar to that found for the oxygen interstitials in the bulk material. This leads to the speculation that, perhaps on the oxide surface, oxygen atoms might also be contributing significantly to this total spin moment. However, from the spin-density distribution [Fig. 9(d)], the largest spin moment is not found on the oxygen atoms but instead on the $\mathrm{Cu}_{\mathrm{CSA}}$ atom, i.e., the fully coordinated $\mathrm{Cu}$ atom in a slightly distorted $\mathrm{O}-\mathrm{Cu}-\mathrm{O}$ arrangement. The local spin moment on $\mathrm{Cu}_{\mathrm{CSA}}$ is $0.12 \mu_{B}$, with the next largest moment also on the coordinatively saturated $\mathrm{Cu}$ atom but in the second $\mathrm{O}-\mathrm{Cu}-\mathrm{O}$ trilayer. This is clearly reflected as well in the projected spin-up and spindown surface band structures as shown in Figs. 9(e) and 9(f), respectively. The spin-resolved $\mathrm{Cu} 3 d$ states due to $\mathrm{Cu}_{\mathrm{CSA}}$ are illustrated with dots showing the weight (by dot size) of the contribution to the full surface band structure. It clearly shows that the surface states near the Fermi level are dominated by the $\mathrm{Cu} 3 d$ states due to $\mathrm{Cu}_{\mathrm{CSA}}$ with a larger weight on the fully occupied spin-up states. Notably, copper vacancies behave rather differently in the bulk and on the surface of this oxide.

From the surface free-energy plot (Fig. 8), we have identified that the $\mathrm{Cu}_{\mathrm{CUS}}$ vacancy likes to "cluster" at the surface, i.e., preferring a higher local concentration of vacancies at the surface of $\mathrm{Cu}_{2} \mathrm{O}(111)$. We have tested a possible antiferromagnetic solution by setting up a $(2 \times 1) \mathrm{Cu}_{2} \mathrm{O}(111)$ surface cell and removed two $\mathrm{Cu}_{\mathrm{CUS}}$ from the surface. An antiferromagnetic ordering is set up by enforcing the one defect site to have a starting spin-up magnetic moment while the other having a spin-down magnetic moment. This structure is then self-consistently relaxed to the magnetic ground state. The surface energy of this antiferromagnetic solution is $0.9 \mathrm{meV} / \AA^{2}$ (29 meV per surface unit cell) less favorable than the ferromagnetic solution. Hence, this surface structure could be a likely source of ferromagnetism in lowdimensional structures. 

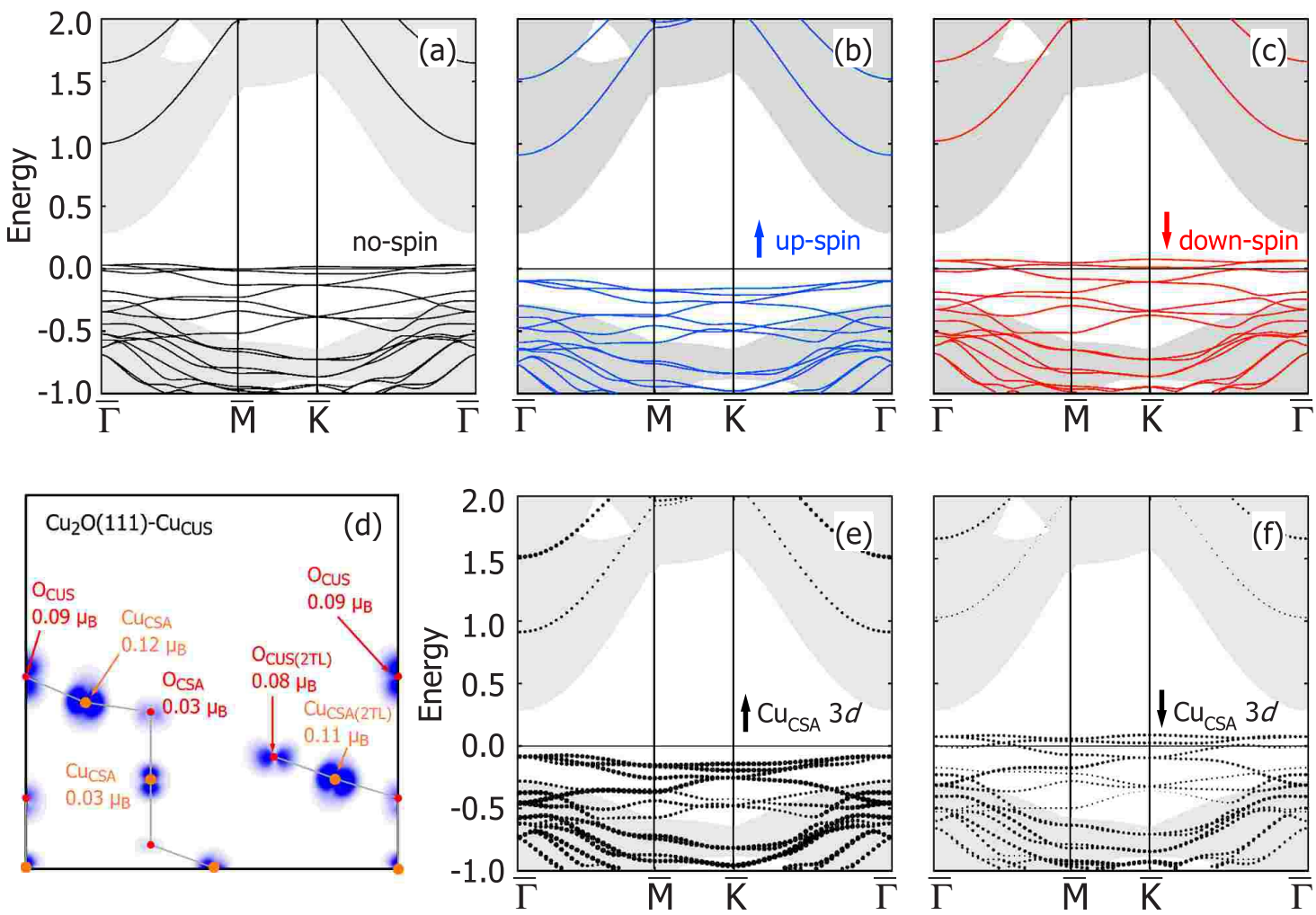

FIG. 9. (Color online) (a) The spin-restricted and [(b) and (c)] spin-unrestricted surface band structure of $\mathrm{Cu}_{2} \mathrm{O}(111)-\mathrm{Cu} \mathrm{Cus}_{\mathrm{Cu}} \cdot \mathrm{Cu} d$ states due to $\mathrm{Cu}_{\mathrm{CSA}}$ are shown in (e) and (f), with dots indicating the weight (by size) of the contribution by $\mathrm{Cu}_{\mathrm{CSA}}$ to the full surface band structure. The projected bulk states are shaded in gray. In (d), the spin densities of the surface atoms and their corresponding magnetic moments are plotted, with the largest moment of $0.12 \mu_{B}$ on $\mathrm{Cu}_{\mathrm{CSA}}$.

Turning to the other low-energy oxide surface, $\mathrm{Cu}_{2} \mathrm{O}(110): \mathrm{CuO}$ is found to be nonmagnetic and thus does not contribute to any surface magnetism. Referring to Fig. 8, we find $\mathrm{Cu}_{2} \mathrm{O}(111)-\mathrm{Cu}_{\mathrm{CuS}}$ to be slightly more favored under oxygen-lean conditions while $\mathrm{Cu}_{2} \mathrm{O}(110): \mathrm{CuO}$ is slightly energetically preferred under oxygen-rich conditions. The crossover point between the two oxide surfaces, in the nonmagnetic case, is shown by the small (downward pointing) black arrow on the right in Fig. 8. However, when considering the ferromagnetic $\mathrm{Cu}_{2} \mathrm{O}(111)-\mathrm{Cu}_{\mathrm{CUS}}$ surface, the crossover point (upward pointing black arrow) now shifts by about $0.25 \mathrm{eV}$ to higher oxygen chemical potential. This translates to about an order-of-magnitude difference in the pressure scale, highlighting the effect of how the magnetic structure could possibly have an impact on relative surface stability.

To provide a more complete picture of the possible contributions to surface magnetism in this oxide, we investigate the magnetic structure of the other high-energy low-index and vicinal surfaces in this work, namely, the $\mathrm{Cu}_{2} \mathrm{O}(100)$ : $\mathrm{O}$, $\mathrm{Cu}_{2} \mathrm{O}(210)$, and $\mathrm{Cu}_{2} \mathrm{O}(311)$ surfaces. We find that all these three surfaces are magnetic as well and with magnetic moments of $0.94 \mu_{B}, 1.99 \mu_{B}$, and $1.97 \mu_{B}$, respectively. We see that the microfaceted vicinal surfaces show a much larger magnetic moment, possibly due to more surface dangling bonds. The spin-resolved total DOS for $\mathrm{Cu}_{2} \mathrm{O}(210)$ and $\mathrm{Cu}_{2} \mathrm{O}(311)$ are shown in Figs. 10(a) and $10(\mathrm{~b})$, respectively. They reflect the generic DOS shape of $\mathrm{Cu}_{2} \mathrm{O}$ :

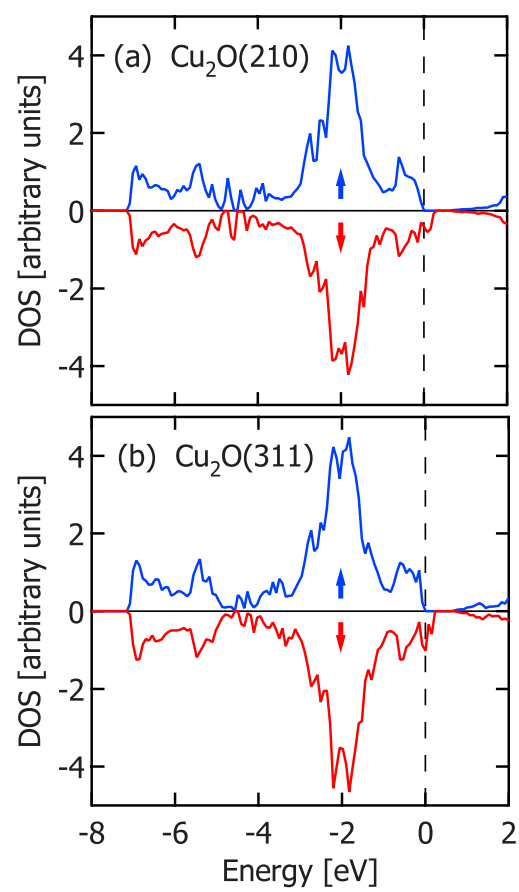

FIG. 10. (Color online) Spin-resolved total density of states of (a) $\mathrm{Cu}_{2} \mathrm{O}(210)$ and (b) $\mathrm{Cu}_{2} \mathrm{O}(311)$. Half-metallic behavior is observed for both high-index surfaces, resulting in a total magnetic spin moment of $1.99 \mu_{B}$ and $1.97 \mu_{B}$, respectively. 
the bonding $\mathrm{O}-\mathrm{Cu}$ states $(-7$ to $-5 \mathrm{eV})$, nonbonding $\mathrm{Cu}$ states $(-4$ to $-1 \mathrm{eV})$, and the antibonding $\mathrm{O}-\mathrm{Cu}$ states $(-1$ to $\sim 0 \mathrm{eV})$. Once again, the spin-up states near the Fermi level are completely filled while the spin-down states are partially occupied, giving rise to a half-metallic character. Although these high-energy surfaces are not thermodynamically stable,kinetic hindrances affecting directional growth rates could possibly promote the existence of such surfaces in reality.

\section{Environment-dependent nanomorphology}

Having investigated both native defects in bulk $\mathrm{Cu}_{2} \mathrm{O}$ and on its surfaces, we have identified bulk oxygen interstitial defects and copper vacancies on $\mathrm{Cu}_{2} \mathrm{O}(111)$, i.e., $\mathrm{Cu}_{2} \mathrm{O}(111)-\mathrm{Cu}_{\mathrm{CUS}}$, to be possible sources of the experimentally observed ferromagnetism in nanoparticles of $\mathrm{Cu}_{2} \mathrm{O} .{ }^{31,32}$ Interestingly, it has also been shown recently that, at room temperature, nanoparticulates $(7-30 \mathrm{~nm})$ (Ref. 30) of otherwise nonmagnetic oxides, e.g., $\mathrm{SnO}_{2}, \mathrm{Al}_{2} \mathrm{O}_{3}$, or even $\mathrm{CeO}_{2}$, are ferromagnetic as well as thin oxide films of similar ceramic oxides. ${ }^{25-27,60}$ These experimental reports claim that nanoparticulate, or thin film, forms of such oxides could possibly create the necessary defects and/or oxygen vacancies, and this may be the main reason for undoped semiconducting and insulating oxides to become ferromagnetic. ${ }^{60}$ In this work, we have shown that perhaps there could be certain truth in this claim.

According to our calculations, we find that ferromagnetism in $\mathrm{Cu}_{2} \mathrm{O}$, which is an otherwise nonmagnetic oxide, is multifarious, i.e., originating from different sources (either the bulk or surface) depending on the gaseous conditions it is in. To illustrate this, we plot the Wulff-Gibbs construction based on our calculated surface energies as a function of the oxygen chemical potential and relate that to temperaturepressure bars for $T=300,400,600$, and $900 \mathrm{~K}$ (shown in Fig. 11). This is done by adopting the same approach of $a b$ initio atomistic thermodynamics as is reported in our previous work. ${ }^{36}$ We express the surface Gibbs free energy as a function of the oxygen chemical potential, and relate the chemical potential of oxygen as a function of pressure and temperature by the ideal-gas equation,

$$
\mu_{\mathrm{O}}=\frac{1}{2}\left[E_{\mathrm{O}_{2}}+\tilde{\mu}_{\mathrm{O}_{2}}\left(p^{0}, T\right)+k_{B} T \ln \left(\frac{p_{\mathrm{O}_{2}}}{p^{0}}\right)\right],
$$

where $p^{0}$ corresponds to standard pressure and $\widetilde{\mu}_{\mathrm{O}_{2}}\left(p^{0}, T\right)$ includes the contributions from rotations and vibrations of the oxygen molecule, as well as the ideal-gas entropy at 1 atm. We refer the reader to Ref. 36 for more details and definitions used in this work. We highlight that the scale for the surface energies is kept fixed for this work, following Ref. 59. In principle, this is not strictly required as the Wulff theorem is only a "theory on shape" rather than size.

Under oxygen-lean conditions (which corresponds to copper-rich conditions), we find that the nanomorphology of the copper-oxide crystal is dominated by the (111) facet. Under such conditions, ferromagnetic behavior originates from the low-energy $\mathrm{Cu}_{2} \mathrm{O}(111)-\mathrm{Cu}_{\mathrm{CuS}}$ surface, rendering it "surface origin." However, with increasing oxygen chemical po-
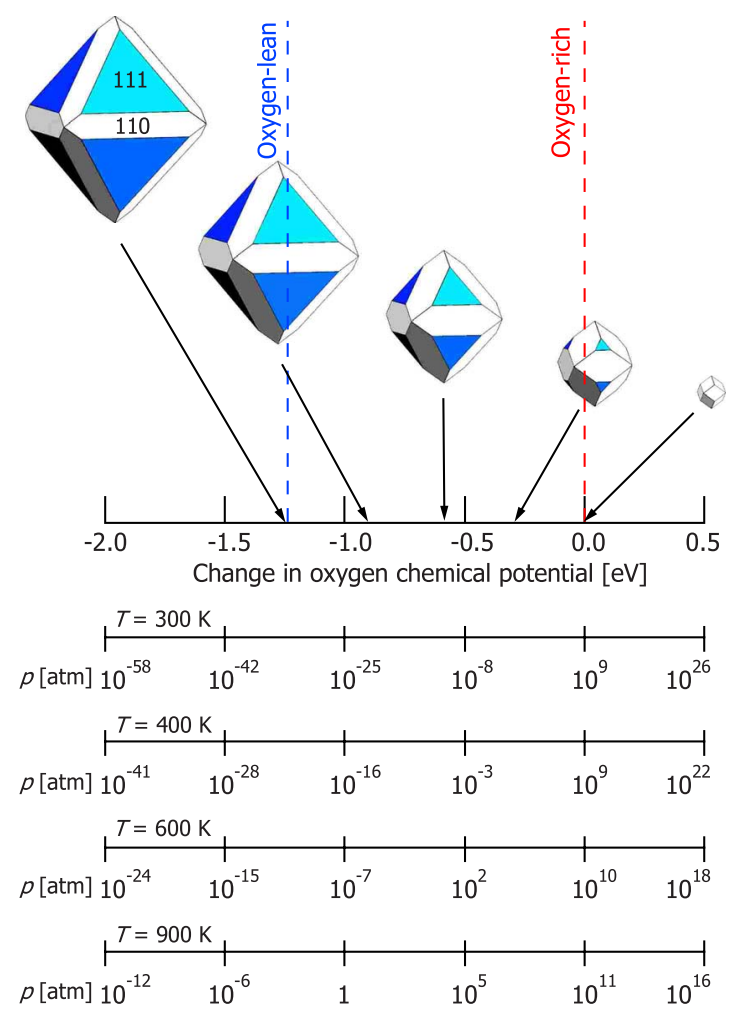

FIG. 11. (Color online) Wulff construction of the $\mathrm{Cu}_{2} \mathrm{O}$ crystal as a function of the change in oxygen chemical potential, $\Delta \mu_{\mathrm{O}}$, from oxygen-lean (left) conditions to oxygen-rich (right) conditions. These shapes are constructed by considering the $(1 \times 1)$ terminations (for both low- and high-index surfaces) and therefore only reflect the relative surface energies of oxide surfaces considered in this work. Corresponding pressure bar lines at $T=300,400$, 600 , and $900 \mathrm{~K}$ are shown. For this work, the scale for the surface energies is kept fixed (Ref. 59). In principle, this is not strictly required as the Wulff theorem is only a theory on shape rather than size.

tential, the (111) facet diminishes and the (110) facet becomes more dominant, also making the crystal more spherical in shape. Under oxygen-rich conditions, the predominant (110) facet is nonmagnetic but high concentrations of oxygen interstitial bulk defects become more energetically favorable, thus rendering the ferromagnetic behavior "bulk origin." From this analysis, it is now not difficult to understand why nanoparticulate, or thin film, forms of metal oxides exhibit ferromagnetism while their bulk counterpart does not. Taking $\mathrm{Cu}_{2} \mathrm{O}$ as an example, we find that the possible crossover point between "surface-origin" and "bulkorigin" ferromagnetism could be around $300-400 \mathrm{~K}$ at about $10^{-3}-10^{-12} \mathrm{~atm}$. If the Curie temperature of these nanostructures exceeds $500 \mathrm{~K}$, perhaps this crossover point could well be at ambient pressures.

\section{CONCLUSIONS}

Native defects in bulk cuprous oxide, $\mathrm{Cu}_{2} \mathrm{O}$, and its surfaces have been studied by using first-principles calculations based on density-functional theory. We considered copper 
and oxygen vacancies, antisites and interstitials, and the copper split-vacancy defect complex. From the calculated formation energies as a function of the change in Fermi level under both copper-rich and oxygen-rich conditions, we found that the simple copper vacancy has the lowest formation energy under both copper-rich and oxygen-rich conditions and it is nonmagnetic. The formation of copper vacancies act as a shallow hole-producer and accounts for the intrinsic $p$-type semiconductivity in $\mathrm{Cu}_{2} \mathrm{O}$. This is further enhanced by the fact that potential hole killers such as the oxygen vacancy is only stable in the neutral charge state, hence not being able to compensate the holes produced. Other potential hole killers such as the copper interstitials and copper antisites have high formation energies and thus have very low defect concentrations. Under oxygen-rich conditions, oxygen interstitials (both at the octahedral and tetrahedral sites) have comparatively low formation energies and are found to couple ferromagnetically with a large magnetic moment of $1.38 \mu_{B}$ and $1.36 \mu_{B}$ at the octahedral and tetrahedral sites, respectively. Considering the possibility of defect formation at the surface of this material, we investigated the relative stability of both low- and high-index copper-oxide surfaces by comparing their surface free energies as a function of the change in oxygen chemical potential. Two low-energy surface structures, namely, the $\mathrm{Cu}_{2} \mathrm{O}(110): \mathrm{CuO}$ under oxygen-rich conditions and $\mathrm{Cu}_{2} \mathrm{O}(111)-\mathrm{Cu}_{\text {CUS }}$ structure under oxygen-lean (or copper-rich) conditions, were identified. Using the calculated surface free energies of the considered low- and highindex oxide surfaces, we performed a Wulff construction and found that the dominance of the (111) surface decreases with increasing oxygen chemical potential. $\mathrm{Cu}_{2} \mathrm{O}(111)-\mathrm{Cu}_{\mathrm{CUS}}$ is calculated to have a total ferromagnetic spin moment of $0.91 \mu_{\mathrm{B}}$ while $\mathrm{Cu}_{2} \mathrm{O}(110): \mathrm{CuO}$ is found to be nonmagnetic. Hence by analyzing the nanomorphology of $\mathrm{Cu}_{2} \mathrm{O}$, we found that surface magnetism, originating from $\mathrm{Cu}_{2} \mathrm{O}(111)-\mathrm{Cu}_{\mathrm{CUS}}$, dominates at low oxygen chemical potential while at high oxygen chemical potential, low-energy bulk defects, namely, oxygen interstitial defects, could possibly induce a ferromagnetic behavior in $\mathrm{Cu}_{2} \mathrm{O}$. This result highlights the complementary role of bulk and surface native magnetic defects under different pressure and temperature conditions, especially at the nanoparticle scale where surface properties could dominate.

\section{ACKNOWLEDGMENTS}

The authors gratefully acknowledge support from the Australian Research Council (ARC), the Australian Partnership for Advanced Computing (APAC), and the Australian Centre for Advanced Computing and Communications (ac3). S.H.W. is grateful to the University of Sydney for support in this work.
*Corresponding author. Present address: Theory Department, FritzHaber-Institut der Max-Planck-Gesellschaft, D-14195 Berlin, Germany. Email: soon@fhi-berlin.mpg.de

${ }^{1}$ A. E. Rakhshani, Solid-State Electron. 29, 7 (1986).

${ }^{2}$ Y. Duan, K. Zhang, and X. Xie, Surf. Sci. 321, L249 (1994).

${ }^{3}$ D. Scarano, S. Bordiga, C. Lamberti, G. Spoto, G. Ricchiardi, A. Zecchina, and C. Otero, Surf. Sci. 411, 272 (1998).

${ }^{4}$ T. J. Huang and D. H. Tsai, Catal. Lett. 87, 173 (2002).

${ }^{5}$ K. Nagase, Y. Zheng, Y. Kodama, and J. Kakuta, J. Catal. 187, 123 (1999).

${ }^{6}$ A. Martínez-Ruiz, M. G. Moreno, and N. Takeuchi, Solid State Sci. 5, 291 (2003).

${ }^{7}$ M. Nolan and S. D. Elliott, Phys. Chem. Chem. Phys. 8, 5350 (2006).

${ }^{8}$ A. F. Wright and J. S. Nelson, J. Appl. Phys. 92, 5849 (2002).

${ }^{9}$ G. G. Jernigan and G. A. Somorjai, J. Catal. 147, 567 (1994).

${ }^{10}$ H. Werner, D. Herein, G. Schulz, U. Wild, and R. Schlögl, Catal. Lett. 49, 109 (1997).

${ }^{11}$ T. Schedel-Niedrig, M. Hävecker, A. Knop-Gericke, and R. Schlögl, Phys. Chem. Chem. Phys. 2, 3473 (2000).

${ }^{12}$ B. White, M. Yin, A. Hall, D. Le, S. Stolbov, T. Rahman, N. Turro, and S. O’Brien, Nano Lett. 6, 2095 (2006).

${ }^{13}$ A. Soon, M. Todorova, B. Delley, and C. Stampfl, Phys. Rev. B 73, 165424 (2006).

${ }^{14}$ H. Raebiger, S. Lany, and A. Zunger, Phys. Rev. B 76, 045209 (2007).

${ }^{15}$ T. Dietl, Nature Mater. 2, 646 (2003).

${ }^{16}$ S. Kuroda, N. Nishizawa, K. Takita, M. Mitome, Y. Bando, K. Osuch, and T. Dietl, Nature Mater. 6, 440 (2007).
${ }^{17}$ M. Bibes and A. Barthélémy, IEEE Trans. Electron Devices 54, 1003 (2007).

${ }^{18}$ J. M. D. Coey, J. Appl. Phys. 97, 10D313 (2005).

${ }^{19}$ S. J. Pearton, W. H. Heo, M. Ivill, D. P. Norton, and T. Steiner, Semicond. Sci. Technol. 19, R59 (2004).

${ }^{20}$ T. Fukumura, H. Toyosaki, and Y. Yamada, Semicond. Sci. Technol. 20, S103 (2005).

${ }^{21}$ S. A. Chambers, T. C. Droubay, C. M. Wang, K. M. Rosso, S. M. Heald, D. A. Schwartz, K. R. Kittilstved, and D. R. Gamelin, Mater. Today 9, 28 (2006).

${ }^{22}$ K. R. Kittilstved, W. K. Liu, and D. R. Gamelin, Nature Mater. 5, 291 (2006).

${ }^{23}$ C. B. Fitzgerald, M. Venkatesan, L. S. Dorneles, R. Gunning, P. Stamenov, J. M. D. Coey, P. A. Stampe, R. J. Kennedy, E. C. Moreira, and U. S. Sias, Phys. Rev. B 74, 115307 (2006).

${ }^{24}$ J. M. D. Coey, Curr. Opin. Solid State Mater. Sci. 10, 83 (2006).

${ }^{25}$ N. H. Hong, J. Sakai, N. Poirot, and V. Brizé, Phys. Rev. B 73, 132404 (2006).

${ }^{26}$ N. H. Hong, J. Sakai, and F. Gervais, J. Magn. Magn. Mater. 316, 214 (2007)

${ }^{27}$ S. Gallego, J. I. Beltrán, J. Cerdá, and M. C. Munoz, J. Phys.: Condens. Matter 17, L451 (2005).

${ }^{28}$ G. Bouzerar and T. Ziman, Phys. Rev. Lett. 96, 207602 (2006).

${ }^{29}$ J. Osorio-Guillén, S. Lany, S. V. Barabash, and A. Zunger, Phys. Rev. B 75, 184421 (2007).

${ }^{30}$ A. Sundaresan, R. Bhargavi, N. Rangarajan, U. Siddesh, and C. N. R. Rao, Phys. Rev. B 74, 161306(R) (2006).

${ }^{31}$ A. Y. Yermakov, M. A. Uimin, A. A. Mysik, V. B. Vykhodets, T. E. Kurennykh, V. I. Sokolov, V. S. Gaviko, N. N. Schegoleva, 
and N. B. Gruzdev, J. Magn. Magn. Mater. 310, 2102 (2007).

${ }^{32}$ V. I. Sokolov, A. Y. Yermakov, M. A. Uimin, A. A. Mysik, V. B. Vykhodets, T. E. Kurennykh, V. S. Gaviko, N. N. Schegoleva, and N. B. Gruzdev, J. Exp. Theor. Phys. 105, 65 (2007).

${ }^{33}$ O. Porat and I. Riess, Solid State Ionics 74, 229 (1994).

${ }^{34}$ O. Porat and I. Riess, Solid State Ionics 81, 29 (1995).

${ }^{35}$ S. Kitashima, A. Kuroda, K. Hirose, M. Senna, A. Y. Yermakov, and M. A. Uimin, J. Alloys Compd. 434-435, 646 (2007).

${ }^{36}$ A. Soon, M. Todorova, B. Delley, and C. Stampfl, Phys. Rev. B 75, 125420 (2007).

${ }^{37}$ J. P. Perdew, K. Burke, and M. Ernzerhof, Phys. Rev. Lett. 77, 3865 (1996).

${ }^{38}$ B. Delley, J. Chem. Phys. 92, 508 (1990).

${ }^{39}$ B. Delley, J. Chem. Phys. 113, 7756 (2000).

${ }^{40}$ Y. Yan and S.-H. Wei, Phys. Status Solidi B 245, 641 (2008).

${ }^{41}$ C. G. Van de Walle and J. Neugebauer, J. Appl. Phys. 95, 3851 (2004).

${ }^{42}$ E. Ruiz, S. Alvarez, P. Alemany, and R. A. Evarestov, Phys. Rev. B 56, 7189 (1997).

${ }^{43}$ C. Kittel, Introduction to Solid State Physics (Wiley, New York, 1996).

${ }^{44}$ W. G. Wickoff, Crystal Structures (Wiley-Interscience, New York, 1960), Vol. 1.

${ }^{45}$ A. F. Wells, Structural Inorganic Chemistry, 5th ed. (Clarendon, Oxford, 1984).

${ }^{46}$ A. Werner and H. D. Hochheimer, Phys. Rev. B 25, 5929 (1982).
${ }^{47}$ D. R. Lide, CRC Handbook of Chemistry and Physics, Internet version 2005 (CRC Press, Boca Raton, FL, 2005).

${ }^{48}$ R. Laskowski, P. Blaha, and K. Schwarz, Phys. Rev. B 67, 075102 (2003).

${ }^{49}$ A. Filippetti and V. Fiorentini, Phys. Rev. B 72, 035128 (2005).

${ }^{50}$ A. Önsten, M. Månsson, T. Claesson, T. Muro, T. Matsushita, T. Nakamura, T. Kinoshita, U. O. Karlsson, and O. Tjernberg, Phys. Rev. B 76, 115127 (2007).

${ }^{51}$ T. Kotani, M. van Schilfgaarde, and S. V. Faleev, Phys. Rev. B 76, 165106 (2007).

${ }^{52}$ J. P. Hu, D. J. Payne, R. G. Egdell, P.-A. Glans, T. Learmonth, K. E. Smith, J. Guo, and N. M. Harrison, Phys. Rev. B 77, 155115 (2008).

${ }^{53}$ A. Soon, T. Söhnel, and H. Idriss, Surf. Sci. 579, 131 (2005).

${ }^{54}$ S. Lany and A. Zunger, Phys. Rev. B 72, 035215 (2005).

${ }^{55}$ G. K. Paul, Y. Nawa, H. Sato, T. Sakurai, and K. Akimoto, Appl. Phys. Lett. 88, 141901 (2006).

${ }^{56}$ L. Fritsche and B. Weimert, Phys. Status Solidi B 208, 287 (1998).

${ }^{57}$ J. L. F. Da Silva, K. Schroeder, and S. Blügel, Phys. Rev. B 69, 245411 (2004).

${ }^{58}$ N. D. Lang and W. Kohn, Phys. Rev. B 3, 1215 (1971).

${ }^{59}$ N. Moll, A. Kley, E. Pehlke, and M. Scheffler, Phys. Rev. B 54, 8844 (1996).

${ }^{60}$ N. H. Hong, A. Barla, J. Sakai, and N. Q. Huong, Phys. Status Solidi C 4, 4461 (2007). 